\title{
Design Tools for Electrochemical Supercapacitors Using Local Absolute Stability Theory
}

\author{
R. Drummond, S. Zhao and S. R. Duncan
}

\begin{abstract}
In this paper, system properties of a physics-based model of a supercapacitor are computed using a local Lyapunov analysis and the solution of linear matrix inequalities. By considering the model as a Lurie system, bounds are computed for the resistance, capacitance, stored and dissipated energies for a charging profile, model stability, maximum reachable set with an input current impulse and the maximum voltage such that the potential difference across the double layer never exceeds the electrolyte overpotential. The bounds computed in this paper will aid the designers of supercapacitors, as the effect on the system properties caused by changes to the physical parameters can now be estimated. The theoretical results predicted by the analysis are compared to experimental data.
\end{abstract}

Index Terms-Supercapacitors , Absolute Stability Theory, Energy Storage, Nonlinear Systems.

\section{INTRODUCTION}

This paper introduces a method for designing energy storage devices known as electric double layer supercapacitors. The proposed approach uses Lyapunov analysis to give bounds on the energy properties of a nonlinear physics-based model of a supercapacitor. The variation of these bounds as functions of the model physical parameters is investigated and compared to experimental results in [48] and [11]. The methods introduced in this paper will help designers, as they directly relate the impact of changes in the physical parameters, such as porosity and diffusion coefficient, to the electrical properties that describe the performance of the device. These properties determine the energy, power and safety of the supercapacitor, which are important for users. Introducing a systematic method for computing these properties, based upon the ideas of systems theory, provides a new methodical approach for supercapacitor design.

Electric double layer supercapacitors, often simply referred to as supercapacitors, can provide high power for applications such as hybrid vehicles, electric load levelling, wind turbines and photo voltaic cells [39]. By storing electrical energy electrostatically on porous electrodes with high specific surface areas, supercapacitors combine the benefits of capacitors (high power densities and low influence of temperature and ageing effects) with batteries

R. Drummond, S. Zhao and S. R. Duncan are with the Department of Engineering Science, University of Oxford, 17 Parks Road, OX1 3PJ Oxford, United Kingdom, Email: \{ross.drummond,shi.zhao,stephen.duncan\}@eng.ox.ac.uk. Work supported by the Engineering and Physical Sciences Research Council. (higher energy densities) giving a 'hybrid' device that has unique energy storage properties [23, 24]. The typical construction of a supercapacitor is shown in Figure 1, with two porous electrodes immersed in an ionic electrolyte, where the electrodes are separated by an electrically insulating separator to prevent short circuiting. Current collectors attached to the outer ends of the electrodes transfer current to and from the system. Charge is transferred through the device by the current and flux of electrolyte ions, with the electrical potential acting as a potential field that drives these fluxes.

The development and application of electrical energy storage devices, such as supercapacitors, has revolutionised many technology sectors including transport, personal electronics and grid storage, and has led to an increased demand on the performance of these devices. Improvements in performance have generally been achieved through advancements in electrochemistry, for example through the design of more advanced electrodes [49]. This approach requires exhaustive electrochemical testing to iterate towards a design with potentially long rest periods between tests to redesign the device. Recently, electrochemical models have been applied to energy storage devices to improve this design process by making predictions about the system properties so that an improved design can be achieved with fewer experiments [34]. Compared to the 'experiment based' optimisation approach based upon experimental testing, this 'model based' approach is faster and considers a wider range of potential designs.

Most existing studies on the design of electrochemical energy storage devices have focused on lithium ion batteries. For example, [45] characterised the degradation of lithium ion batteries using impedance spectroscopy, [27] considered the optimal electrode length and porosity and [14] optimized the utilisation of the active material in a lithium ion electrode. The optimal grading of electrode porosity for lithium ion batteries was considered in $[15,6]$. Physical models of lithium ion batteries have also been used to develop optimal charging profiles both for reducing mechanical stress in the battery [43] and for reducing ageing effects [41]. In [42], the impact of the electrode design on capacity fade is investigated. Optimising physical model parameters for specific charging profiles to maximise the stored energy [7] and to minimise the Ohmic losses [33] have also been developed. By contrast, the design of supercapacitors has received less attention, with recent results including [22] and [16]. In [37], an equivalent circuit model was used for the design of supercapacitors and 


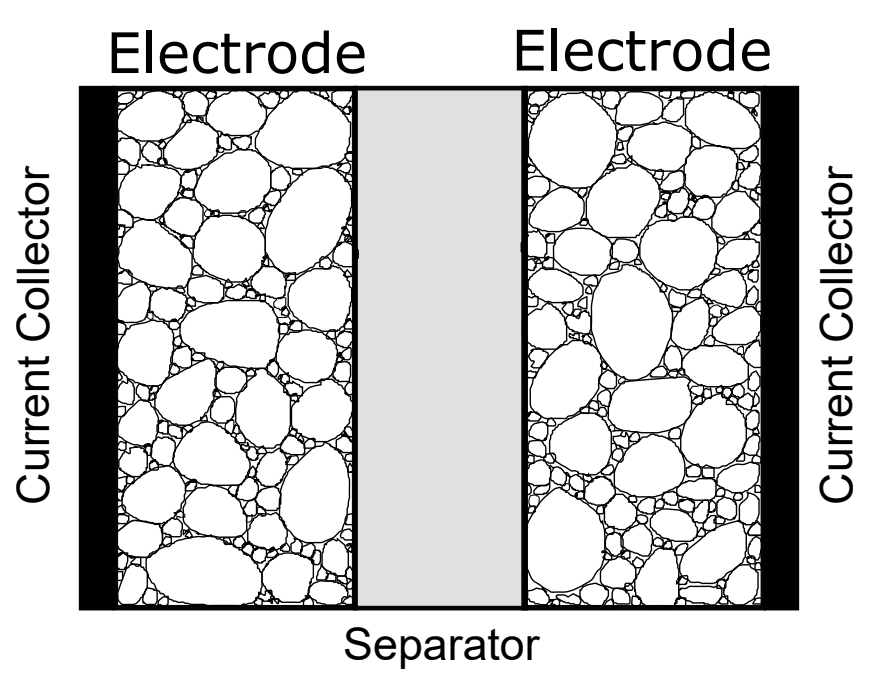

Figure 1: The standard construction of a supercapacitor.

the heat management of a supercapacitor for an electric vehicle application was outlined in [1]. These works show the growing importance that models, optimisation and systems analysis have in the field of energy storage.

One of the drawbacks of model based design is that the quality of the predictions depend upon the quality of the model. For this reason, a "good" model that effectively describes the device dynamics for a range of operating regimes is required. There are two main types of supercapacitor models: equivalent circuit (EC) and physics based (PB) models. EC models are fast and simple to implement, but they are local models and their model states have little physical meaning [51]. Physics based models use conservation and diffusion equations to describe the movement of ions due to electrical forcing. As these models are described by partial differential equations, they are in general more complex to solve than EC models, but they have the advantage that the model states have a physical interpretation [12]. This means that PB models are generally more descriptive than EC models, since they provide predictions of the physical state of the device. Additional physical relationships of interest, such as temperature dependence [8], can also be readily incorporated into a PB model, although the extra information provided by these models comes at the expense of increased model complexity. Since the goal of this paper is to relate the supercapacitor's physical parameters to its performance, only PB models are considered.

Several physics-based models of supercapacitors have already been developed in the literature. The PDE transport equations and boundary conditions for a supercapacitor were introduced in [48] and the implementation of this model was studied in $[2,10]$. A reduced order model of the model presented in [48] was developed in [38] and an analytical solution for constant current and EIS charging was described in [40] for a similar PB model. Models capable of capturing additional physical phenomena, including temperature [8] and self-discharge [31], have also been developed. In the related field of lithium ion batteries, the Newman model [30] has become widely adopted. This model is more complicated than most equivalent supercapacitor models such as [48], but it is based on many similar relationships including ion transport and Ohm's law [3].

In this paper, methods from systems analysis and control theory $[21,4]$ are applied to a PB supercapacitor model, whose PDEs are discretised in space using the spectral collocation method [10]. These methods give bounds for the following system properties of the model:

- Model stability, characterising the limits of the model for safe use.

- Estimates of reachable sets for the states describing the ionic concentration and electropotential. These reachable sets are also related to safety as they characterise the maximum deviation in the states due to a unit impulse in energy via the current.

- Dissipated and stored energies for a charging profile to give more accurate predictions for the state-of-charge of the supercapacitor, which is one of the key design metrics for an electrical energy storage device.

- Gains from current to voltage that describe the capacitance and the resistance.

- Safe maximum voltages such that electrolyte degradation is avoided. This property impacts the maximum stored energy of a supercapacitor which is proportional to the maximum voltage squared.

The bounds are computed using absolute stability theory and the solution of optimisation problems involving linear matrix inequalities (LMIs) and semi-definite programming. Absolute stability theory is a classical control problem, which considers the stability properties of nonlinear systems that can be expressed as the feedback interconnection of a linear system with a nonlinearity that is bounded around the origin by a cone [21]. A key benefit of the theory is that the results are applicable to all nonlinearities satisfying the cone condition. As such, it allows a wide class of nonlinear systems to be analysed using the well understood concepts of feedback developed for linear systems, with a popular application being saturated systems [18]. Numerous solutions have been proposed for the absolute stability problem, with these solutions in general being posed either in the frequency domain or in the time domain. Frequency domain results include some of the original results of Kalman, Popov and Yakubovich, who showed that if a "multiplier" could be found that shifted the phase of the transfer function of the linear system in a suitable manner, then it implied the existence of a particular Lyapunov function and hence stability could be verified [35]. This result sparked considerable attention with numerous different forms of multipliers then being proposed [50]. Time domain solutions to the absolute stability problem generally involve the search for suitable Lyapunov functions and for particular classes of such functions, this search can be solved numerically using fast semi-definite programming solvers [4].

Generally speaking, the solutions to absolute stability 


\begin{tabular}{|c|c|}
\hline Symbol & Definition \\
\hline \multicolumn{2}{|r|}{ Space and Time } \\
\hline$t$ & Time (s) \\
\hline$\chi$ & Spatial co-ordinate $(\mathrm{m})$ \\
\hline \multicolumn{2}{|r|}{ Model States } \\
\hline$c(\chi, t)$ & Ionic concentration $\left(\mathrm{mol} \mathrm{m}^{-3}\right)$ \\
\hline$\phi_{s}(\chi, t)$ & Potential in the electrode $(\mathrm{V})$ \\
\hline$\phi_{e}(\chi, t)$ & Potential in the electrolyte (V) \\
\hline \multicolumn{2}{|r|}{ Model Parameters } \\
\hline$\epsilon$ & Porosity coefficient \\
\hline$D$ & Diffusion coefficient $\left(\mathrm{m}^{2} \mathrm{~s}^{-1}\right)$ \\
\hline$a C$ & Specific Capacitance $\left(\mathrm{F} \mathrm{m}^{-2}\right)$ \\
\hline$F$ & Faraday constant $\left(\mathrm{A} \mathrm{mol}^{-1}\right)$ \\
\hline$t_{+}$and $t_{-}$ & Transference numbers satisfying $t_{+}+t_{-}=1$ \\
\hline & Change in ion surface concentration \\
\hline & with change in electrode surface charge $q$ \\
\hline$\sigma$ & Electrode conductivity $\left(\mathrm{S} \mathrm{m}^{-1}\right)$ \\
\hline$\kappa$ & Electrolyte conductivity $\left(\mathrm{S} \mathrm{m}^{-1}\right)$ \\
\hline$R$ & Universal gas constant $\left(\mathrm{J} \mathrm{K}^{-1} \mathrm{~mol}^{-1}\right)$ \\
\hline$T$ & Temperature $(\mathrm{K})$ \\
\hline$f=\frac{F}{R T}$ & A coefficient \\
\hline$I(t)$ & Current (A) \\
\hline$S A$ & Electrode surface area $\left(\mathrm{m}^{2}\right)$ \\
\hline$L_{\text {elec }}$ & Length of the electrodes $(\mathrm{m})$ \\
\hline$L_{s e p}$ & Length of the separator $(\mathrm{m})$ \\
\hline$L=2 L_{e l e c}+L_{s e p}$ & Total Length of the device $(\mathrm{m})$ \\
\hline \multicolumn{2}{|r|}{ Model Input/Output } \\
\hline$i(t)=\frac{I(t)}{S A}$ & Current density $\left(\mathrm{A} \mathrm{m}^{-2}\right)$ \\
\hline$v(t)$ & Voltage (V) \\
\hline
\end{tabular}

Table I: List of symbols that define the electrochemical supercapacitor model.

that are posed in the frequency domain tend to be less conservative than those posed in the time domain. However, the time domain conditions have two main advantages. The first is that they permit a local analysis as they exploit the geometric conditions of the positively invariant level sets of the Lyapunov functions. Secondly, they can be extended to the case of the feedback being multi-input/multi-output (MIMO). Even though MIMO frequency domain stability methods have been developed, it is less intuitive to work with these methods compared to Lyapunov functions. In this work, a local analysis of a MIMO nonlinear feedback system that describes the electrochemistry of a supercapacitor is considered, so the time domain approach is used. Local absolute stability theory was developed in $[17,5]$ for an application to saturating systems where it was shown that tighter results could be obtained than those obtained for a global analysis. A recent extension of these results was developed in [46] for the case of the nonlinearity being slope-restricted.

The underlying theme of this paper is the characterisation of electrical properties of nonlinear supercapacitor models using local absolute stability theory. The technical contributions extend existing results on supercapacitor model analysis because the nonlinearities are treated in a rigorous manner, with the results also representing one of the few applications of local absolute stability theory to physical systems. The analysis given here manipulates the model equations in such a way as to allow them to be analysed using systems theory. The main novelty lies with the application of the theoretical tools of stability theory to a physical system so as to reveal its natures in a manner that incorporates the nonlinearity. As the stability theory results and the model have been developed previously, the analysis presented here is in a rather compact form, with a more extensive analysis given in the accompanying references.

The paper is structured as follows; firstly the PB model equations will be introduced in Section II. In Section III, semi-definite programs are proposed for bounding the system properties of the supercapacitor model with the theoretical results being compared to experimental data.

\section{Notation}

The symbols used to characterise the electrochemical supercapacitor model are given in Table I. Bold font is used to denote vectors and matrices. The solutions to the PDEs are non-bold. For the analysis of Lurie-Postnikov system, the notation of [4] is adopted. The $\mathcal{L}_{2}$ norm of a signal $\theta$ is

$$
\|\theta\|_{2}=\sqrt{\int_{0}^{\infty} \theta(t)^{T} \theta(t) d t .}
$$

The sublevel sets of a positive definite Lyapunov function $V(\theta(t))>0$ are denoted $\mathcal{E}(V, \alpha)=\{\theta(t): V(\theta(t)) \leq \alpha\}$. The time derivative of a function is often referred to as $\dot{V}(\theta(t))=\frac{d V(\theta(t))}{d t}$. The identity matrix is denoted $\boldsymbol{I}$ and the vector containing ones is $\mathbf{1}$. In a slight abuse of notation, we may use $\boldsymbol{\theta}$ to refer to the evaluation of a finite dimensional signal at time $t$, i.e. $\boldsymbol{\theta}(t)$, when it is clear from the context.

\section{Electrochemical Supercapacitor Model}

The non-linear PB supercapacitor model that forms the basis of the proposed design analysis will first be introduced. The particular PB model studied in this paper was developed in [10], which is a reformulation of the partial differential algebraic equation system outlined in [48]. The supercapacitor is treated as a $1 \mathrm{D}$ system, since $3 \mathrm{D}$ effects have been shown to be insignificant at the length scale of the model [2], although, they are important for atomistic level models. The two model domains, the separator and the electrodes, are characterised by the supercapacitor construction, which is shown in Figure 1. The symbols used to define the model are given in Table I and the parameter values for two supercapacitors of the simulation results of Section III are given in Table II.

The three model equations from [10] that respectively describe electrolyte diffusion, double layer charging and a nonlinear concentration dependent version of Kirchoff's 


\begin{tabular}{|c|c|c|c|}
\hline Parameter & SAFT America & Maxwell Technologies & Units \\
\hline \multicolumn{4}{|c|}{ Global Parameters } \\
\hline$T$ & 298 & 298 & $\mathrm{~K}$ \\
$t_{+}$ & 0.55 & 0.75 & \\
$\frac{d q_{+}}{d q}=\frac{d q_{-}}{d q}$ & -0.5 & -0.5 & $\mathrm{~mol} \mathrm{~m}^{-3}$ \\
$c_{0}$ & 930 & 500 & $\mathrm{~S} \mathrm{~m}^{-1}$ \\
\hline \multicolumn{4}{|c|}{ Electrode Parameters } \\
\hline$\kappa$ & 0.020 & 0.026 & $\mathrm{~m}^{2} \mathrm{~s}^{-1}$ \\
$D$ & $2.09 \times 10^{-12}$ & $3.67 \times 10^{-11}$ & $\mathrm{~S} \mathrm{~m}^{-1}$ \\
$\epsilon$ & 0.67 & 0.67 & $\mathrm{~F} \mathrm{~m}^{-2}$ \\
$\sigma$ & 0.052 & $1.14 \times 10^{-} 4$ & $\mathrm{~m}^{2}$ \\
$a C$ & $42 \times 10^{6}$ & $1.87 \times 10^{5}$ & $\mathrm{~m}^{2}$ \\
$L_{\text {elec }}$ & $50 \times 10^{-6}$ & $25 \times 10^{-6}$ & $\mathrm{~S} \mathrm{~m}^{-1}$ \\
$S A$ & 2.747 & 2 & $\mathrm{~m}^{2} \mathrm{~s}^{-1}$ \\
\hline \multicolumn{4}{|c}{ Separator Parameters } \\
\hline$\kappa$ & 0.031 & $5.78 \times 10^{-5}$ & $\mathrm{~m}^{-12}$ \\
$D$ & $3.34 \times 10^{-12}$ & $8.19 \times 10^{-15}$ & 0.6 \\
$L_{\text {sep }}$ & $25 \times 10^{-6}$ & $10 \times 10^{-6}$ & \\
\hline
\end{tabular}

Table II: Parameters for the supercapacitor model. The parameters for the SAFT America and the Maxwell Technologies supercapacitors were respectively obtained from [48] and [11].

current law are

$$
\begin{gathered}
\epsilon \frac{\partial c(\chi, t)}{\partial t}=D \frac{\partial^{2} c(\chi, t)}{\partial \chi^{2}} \\
\quad-\frac{a C}{F}\left(t_{-} \frac{d q_{+}}{d q}+t_{+} \frac{d q_{-}}{d q}\right) \frac{\partial\left(\phi_{s}(\chi, t)-\phi_{e}(\chi, t)\right)}{\partial t} \\
a C \frac{\partial\left(\phi_{s}(\chi, t)-\phi_{e}(\chi, t)\right)}{\partial t}=\sigma \frac{\partial^{2} \phi_{s}(\chi, t)}{\partial \chi^{2}}, \\
0=\frac{\kappa R T}{F}\left(t_{+}-t_{-}\right) \frac{\partial}{\partial \chi} \ln (c(\chi, t))+i(t) \\
\quad+\sigma \frac{\partial\left(\phi_{s}(\chi, t)-\phi_{e}(\chi, t)\right)}{\partial \chi}+\left(\kappa \frac{\partial}{\partial \chi}+\sigma \frac{\partial}{\partial \chi}\right) \phi_{e}(\chi, t) .
\end{gathered}
$$

The model states are the volume averaged ionic concentration $c(\chi, t)$, the electropotential in the solid electrodes $\phi_{s}(\chi, t)$ and the electropotential in the electrolyte $\phi_{e}(\chi, t)$. The model input is the current density $i$ and the model output is the voltage $v$ which is the difference in electropotentials between the two current collectors

$$
v(t)=\phi_{s}(0, t)-\phi_{s}(L, t)
$$

The supercapacitors considered are symmetric with $L_{e l e c}$ being the same for both electrodes. Equation (2a) describes the diffusion of the electrolyte ions under a forcing term due to the potential difference between the solid electrode and the liquid electrolyte, known as the double layer potential. The second model equation (2b) describes the transient charging/discharging response of the double layer potential driven by molar fluxes [48]. The third equation (2c) is a version of Kirchoff's current law for the currents in the electrode and electrolyte phases, which incorporates the concentration dependency of the electrolyte current [48]. The model boundary conditions

\begin{tabular}{|c|c|}
\hline \multicolumn{2}{|c|}{ Boundary Conditions } \\
\hline Current Collector/Electrode & Electrode/Separator \\
\hline$\frac{\partial c(\chi, t)}{\partial \chi}=0$ & $\left.D \frac{\partial c(\chi, t)}{\partial \chi}\right|_{\text {elec }}=\left.D \frac{\partial c(\chi, t)}{\partial \chi}\right|_{\text {sep }}$ \\
\hline$\frac{\partial \phi_{s}(\chi, t)}{\partial \chi}=-\frac{i}{\sigma}$, & $\frac{\partial \phi_{s}(\chi, t)}{\partial \chi}=0$ \\
$\phi_{s}(0, t)=0$ & {$\left[\kappa \frac{\partial \phi_{e}}{\partial \chi}+\kappa\left(\frac{t_{+}-t_{-}}{f}\right) \frac{\partial \ln (c)}{\partial \chi}\right]_{e l e c}$} \\
\hline$\frac{\partial \phi_{e}}{\partial \chi}=0$ & $=\left[\kappa \frac{\partial \phi_{e}}{\partial \chi}+\kappa\left(\frac{t_{+}-t_{-}}{f}\right) t \frac{\partial \ln (c)}{\partial \chi}\right]_{s e p}$ \\
\hline
\end{tabular}

Table III: Boundary conditions for the electrochemical supercapacitor model. These conditions are derived from those given in [48]. The boundaries relate to the regions defined in Figure 1. The subscripts elec and sep respectively relate to derivatives pointing into the electrode and the separator using the notation of [48].

are given by Table III and are obtained from [48]. These boundary conditions can be summarised as conserving the total amount of ionic concentration in the device with all current being transferred through the electrodes at the current collectors and all current being transferred by the electrolyte in the separator. Also, a reference potential is set for the solid electrode potential at $\chi=0$.

\section{A. Model Reformulation}

In order to convert the logarithmic nonlinearity of (2c) into one that is sector bounded and applicable for absolute stability analysis [21], the concentration is defined as $c(\chi, t)=c_{0}+\tilde{c}(\chi, t)$, where $c_{0}$ is the equilibrium concentration, which is constant with respect to $\chi$ and $\tilde{c}(\chi, t)$ is the concentration deviation. This transformation changes $(2 \mathrm{a})$ and $(2 \mathrm{c})$ into

$$
\begin{aligned}
\epsilon \frac{\partial \tilde{c}(\chi, t)}{\partial t}= & D \frac{\partial^{2} \tilde{c}(\chi, t)}{\partial \chi^{2}} \\
& -\frac{a C}{F}\left(t_{-} \frac{d q_{+}}{d q}+t_{+} \frac{d q_{-}}{d q}\right) \frac{\partial\left(\phi_{s}(\chi, t)-\phi_{e}(\chi, t)\right)}{\partial t} \\
0= & \frac{\kappa R T}{F}\left(t_{+}-t_{-}\right) \frac{\partial}{\partial \chi} \ln \left(1+\frac{\tilde{c}(\chi, t)}{c_{0}}\right)+i \\
& +\sigma \frac{\partial\left(\phi_{s}(\chi, t)-\phi_{e}(\chi, t)\right)}{\partial \chi}+\left(\kappa \frac{\partial}{\partial \chi}+\sigma \frac{\partial}{\partial \chi}\right) \phi_{e}(\chi, t)
\end{aligned}
$$

since $\ln (c(\chi, t))=\ln \left(1+\tilde{c}(\chi, t) / c_{0}\right)+\ln \left(c_{0}\right)$ and

$$
\frac{\partial c_{0}}{\partial t}=\frac{\partial c_{0}}{\partial \chi}=\frac{\partial^{2} c_{0}}{\partial \chi^{2}}=\frac{\partial \ln \left(c_{0}\right)}{\partial \chi}=0 .
$$

The state-space form of the electrode dynamics are given in $(6 a)$ while those of the separator are given in $(6 \mathrm{~b})$, with the differance being because $\phi_{s}(\chi, t)$ does not exist in the separator as it an electrical insulator.

The electrochemical assumptions of the model are outlined in detail in [48]. To summarise, the main model assumptions are that porous electrode theory is employed to describe diffusion in the electrodes [29] and dilute solution theory is used to relax the concentration dependence of the electrolyte parameters [28]. Capacitance is also treated 


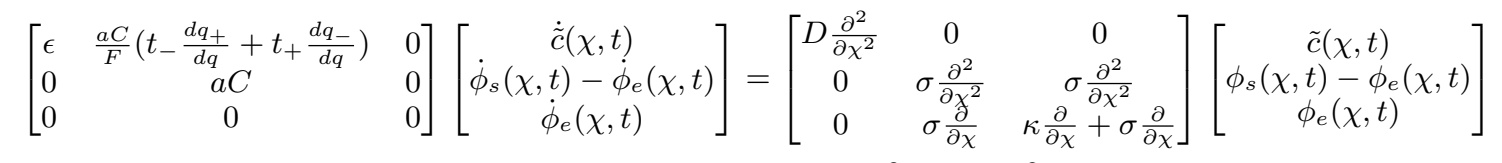

$$
\begin{aligned}
& +\left[\begin{array}{cc}
0 & 0 \\
\kappa\left(\frac{t_{+}-t_{-}}{f}\right) \frac{\partial}{\partial \chi} & 1
\end{array}\right]\left[\begin{array}{c}
\ln \left(1+\frac{\tilde{c}(\chi, t)}{c_{0}}\right) \\
i(t)
\end{array}\right]
\end{aligned}
$$

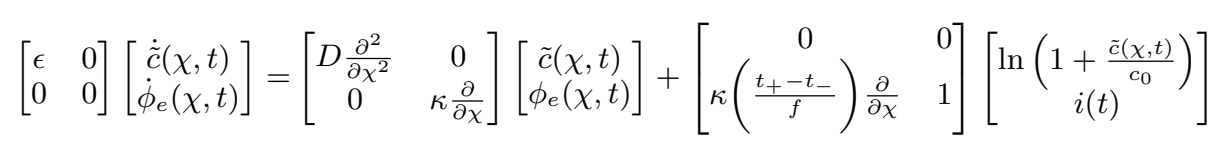

$$
\left[\begin{array}{ccc}
\epsilon \boldsymbol{I} & \frac{a C}{F}\left(t_{-} \frac{d q_{+}}{d q}+t_{+} \frac{d q_{-}}{d q}\right) \boldsymbol{I} & 0 \\
0 & a C \boldsymbol{I} & 0 \\
0 & 0 & 0
\end{array}\right]\left[\begin{array}{c}
\dot{\tilde{\boldsymbol{c}}} \\
\dot{\boldsymbol{\phi}_{s}-\dot{\boldsymbol{\phi}}_{e}} \\
\dot{\boldsymbol{\phi}}_{e}
\end{array}\right]=\left[\begin{array}{ccc}
D \hat{\mathbf{D}}_{c}^{2} & 0 & 0 \\
0 & \sigma \hat{\mathbf{D}}_{\phi_{s}}^{2} & \sigma \hat{\mathbf{D}}_{\phi_{s}}^{2} \\
0 & \sigma \hat{\mathbf{D}}_{\phi_{s}} & \kappa \hat{\mathbf{D}}_{\phi_{e}}+\sigma \hat{\mathbf{D}}_{\phi_{s}}
\end{array}\right]\left[\begin{array}{c}
\tilde{\boldsymbol{c}} \\
\phi_{\boldsymbol{s}}-\phi_{\mathbf{e}} \\
\phi_{\boldsymbol{e}}
\end{array}\right]+\left[\begin{array}{cc}
0 & 0 \\
0 & 0 \\
\kappa\left(\frac{t_{+}-t_{-}}{f}\right) \hat{\mathbf{D}}_{\ln c} & \mathbf{1}
\end{array}\right]\left[\begin{array}{c}
\ln \left(1+\frac{\tilde{\boldsymbol{c}}}{c_{\mathbf{0}}}\right) \\
i(t)
\end{array}\right]
$$

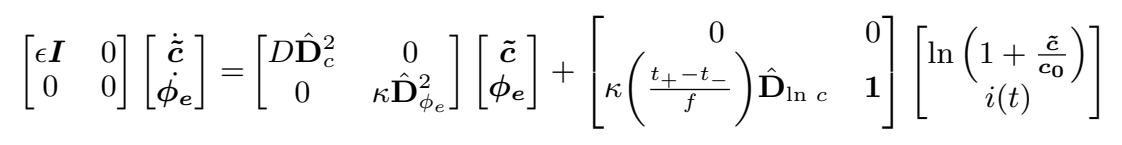

as a fixed parameter, although it has been shown experimentally that it varies during a charge [25]. Additional phenomena, such as varying temperature across the device and leakage current, have not been included. However, the model has been shown to accurately simulate experimental data $[48,11]$, whilst being in a form that is simple enough to be amenable for system analysis.

Nonlinear partial differential algebraic equation systems, such as (6), are typically implemented with spatial discretisation as they are, in general, too complex to be solved analytically. The approximation of an infinite dimensional system by a finite dimensional realisation introduces some level of error, but the discretised system was shown to be accurate when compared against experimental results [10]. Due to space constraints, the spatial discretisation procedure is not detailed here and we refer the reader to [10] where the discretised model is formed. In this paper, a spectral collocation approach with Chebyshev polynomial basis functions is used with the subdomains of the problem (the electrodes and separator) being connected via patching so as to have a locally smooth solution [44]. The reference [44] gives an overview of this discretisation procedure. Applying the techniques of [44], as in [10], to the PDE model (6) results in the spatial differential operators involving $\partial / \partial \chi$ terms being approximated by the differentiation matrix $\hat{\mathbf{D}}_{s}$, where the subscript $s \in\left\{c, \phi_{s}, \phi_{e}, \ln c\right\}$ indicates that the matrix accounts for the boundary terms of $s$ when patching is considered [44]. The use of a spectral collocation discretisation means that a relatively low number (of the order of 20) spatial discretisation elements can give an accurate solution [10], with this being important for the resulting optimisation problems of Section III, as the complexity of these results grows rapidly with the size of the system.

The discretised versions of the supercapacitor model equations (6) for the electrode and separator domains are respectively given in (7a) and(7b). Equation (7b) can be embedded into the structure of ( $7 \mathrm{a})$ by expanding the state dimension and as such only the form from (7a) will be analysed in the following analysis. The discretised model output is given by

$$
v(t)=\left[\begin{array}{ll}
\mathbf{0} & \tilde{C}
\end{array}\right]\left[\begin{array}{c}
\tilde{\boldsymbol{c}} \\
\phi_{s}-\phi_{e}
\end{array}\right]+\tilde{C} \phi_{e}+\tilde{D}_{i} i(t)
$$

when the boundary conditions on $\phi_{s}(\chi, t)$ are considered [48].

An expression for the algebraic vairable $\phi_{\boldsymbol{e}}$ can be obtained by solving the algebraic equation of (7a)

$$
\begin{aligned}
& \phi_{\boldsymbol{e}}=-\left(\kappa \hat{\mathbf{D}}_{\phi_{e}}+\sigma \hat{\mathbf{D}}_{\phi_{s}}\right)^{-1} \\
& \left(\kappa\left(\frac{t_{+}-t_{-}}{f}\right) \hat{\mathbf{D}}_{\ln c} \ln \left(1+\frac{\tilde{\boldsymbol{c}}}{\boldsymbol{c}_{\mathbf{0}}}\right)+\sigma \hat{\mathbf{D}}_{\phi_{s}}\left(\boldsymbol{\phi}_{\boldsymbol{s}}-\boldsymbol{\phi}_{\boldsymbol{e}}\right)+i(t)\right) .
\end{aligned}
$$

Solving (9) requires setting a reference potential as it is only dependent upon the potential difference $\phi_{s}-\phi_{e}$. In the PB model (2), only potential gradients, not the potential values themselves, are used to describe the system dynamics and so an arbitrary reference can be set. For this paper, the potential in the electrode at one of the current collectors is set as the reference. Using the expression for $\phi_{\boldsymbol{e}}$ in (9) means that (7a) can be expressed as an ordinary 


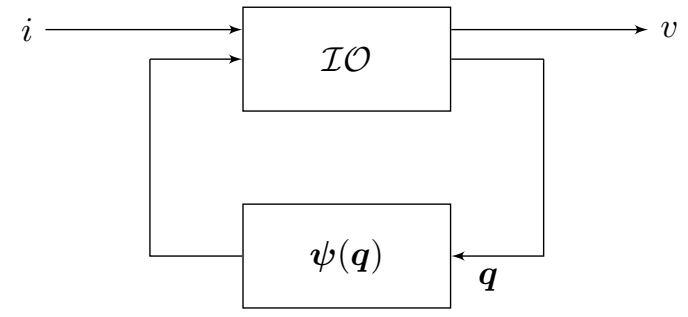

Figure 2: The supercapacitor model expressed in a feedback loop with the logarithmic nonlinearity being treated as an input.

differential equation (ODE) with structure

$$
\begin{array}{r}
{\left[\begin{array}{cc}
M_{11} & M_{12} \\
\mathbf{0} & \boldsymbol{M}_{22}
\end{array}\right]\left[\begin{array}{c}
\dot{\tilde{\boldsymbol{c}}} \\
\dot{\phi}_{s}-\dot{\phi}_{e}
\end{array}\right]=\left[\begin{array}{cc}
\hat{\boldsymbol{A}}_{11} & \mathbf{0} \\
\mathbf{0} & \hat{\boldsymbol{A}}_{\mathbf{2} 2}
\end{array}\right]\left[\begin{array}{c}
\tilde{\boldsymbol{c}} \\
\boldsymbol{\phi}_{\boldsymbol{s}}-\boldsymbol{\phi}_{\boldsymbol{e}}
\end{array}\right]} \\
+\left[\begin{array}{c}
0 \\
\hat{\boldsymbol{B}}_{\mathbf{1}}
\end{array}\right] \ln \left(1+\frac{\tilde{\boldsymbol{c}}}{\boldsymbol{c}_{\mathbf{0}}}\right)+\left[\begin{array}{c}
\mathbf{0} \\
\hat{\boldsymbol{B}}_{\mathbf{2}}
\end{array}\right] i(t) \\
v(t)=\left[\begin{array}{ll}
\mathbf{0} & \hat{\boldsymbol{C}}
\end{array}\right]\left[\begin{array}{c}
\tilde{\boldsymbol{c}} \\
\boldsymbol{\phi}_{\boldsymbol{s}}-\boldsymbol{\phi}_{\boldsymbol{e}}
\end{array}\right]+\hat{\mathbf{D}} \ln \left(1+\frac{\tilde{\boldsymbol{c}}}{\boldsymbol{c}_{\mathbf{0}}}\right)+\hat{D}_{i} i(t) .
\end{array}
$$

The matrices defining the dynamical equation (10a) are given in the appendix with the matrices defining the output (10b) being obtained from the discretised version of the boundary conditions on $\phi_{s}(\chi, t)$ from Table III.

The dynamical equation (10a) can be expressed as

$$
\boldsymbol{M} \dot{\boldsymbol{x}}=\hat{\boldsymbol{A}} \boldsymbol{x}+\hat{\boldsymbol{B}} \ln \left(1+\frac{\tilde{\boldsymbol{c}}}{\boldsymbol{c}_{\mathbf{0}}}\right)+\hat{\boldsymbol{B}}_{\boldsymbol{i}} i(t)
$$

with state vector $\boldsymbol{x}:=\left[\tilde{\boldsymbol{c}}^{T}, \boldsymbol{\phi}_{\boldsymbol{s}}^{T}-\boldsymbol{\phi}_{\boldsymbol{e}}^{T}\right]^{T} \in \mathbb{R}^{n}$ and inverting the "mass" matrix $\boldsymbol{M}$ leads to

$$
\begin{aligned}
\dot{\boldsymbol{x}} & =\hat{\boldsymbol{A}}_{\boldsymbol{M}} \boldsymbol{x}+\hat{\boldsymbol{B}}_{\boldsymbol{M}} \ln \left(1+\frac{\tilde{\boldsymbol{c}}}{\boldsymbol{c}_{\mathbf{0}}}\right)+\hat{\boldsymbol{B}}_{\boldsymbol{i}, \boldsymbol{M}} i(t), \\
v(t) & =\boldsymbol{C} \tilde{\boldsymbol{x}}+\hat{\boldsymbol{D}} \ln \left(1+\frac{\tilde{\boldsymbol{c}}}{\boldsymbol{c}_{\mathbf{0}}}\right)+\hat{\boldsymbol{D}}_{\boldsymbol{i}} i(t) .
\end{aligned}
$$

Matrices $\boldsymbol{M}_{\mathbf{1 1}}, \boldsymbol{M}_{\mathbf{2 2}}$ are diagonal and full rank and hence $\boldsymbol{M}$ is invertible. This system has the feedback structure of Figure 2 where $\boldsymbol{q}=\tilde{\boldsymbol{c}} / c_{0}=\boldsymbol{C}_{\boldsymbol{c}} \boldsymbol{x}, \boldsymbol{\psi}(\boldsymbol{q})=\ln \left(1+\tilde{\boldsymbol{c}} / \boldsymbol{c}_{\mathbf{0}}\right)$ and $\mathcal{I O}$ is the mapping $\{\boldsymbol{\psi}(\boldsymbol{q}), i\} \rightarrow\{\boldsymbol{q}, v\}$.

\section{B. Integrators}

Because the supercapacitor is an energy storage device that absorbs charge supplied by the current, its dynamics include $n_{i}$ integrator states whose trajectories are the integral of the current. For stability analysis, the presence of these integrators states is problematic as they do not exist in the $\mathcal{L}_{2}$ Hilbert space. The absolute stability of such critically stable systems was considered [36] and in [20] using a non-local Zames-Falb multiplier approach where the unbounded integrator dynamics are handled by encapsulating the integrator via a transformation of the system feedback loop. This encapsulation operation is not required for the analysis of the supercapacitor model as the concentration deviation $\tilde{\boldsymbol{c}} / c_{0}=\boldsymbol{q}$ was independent of the integral of the current for both supercapacitors analysed in this paper, which are described by the parameters in Table II.

To show this independence, the eigen-decomposition of the system (12) was taken by defining $\tilde{\boldsymbol{x}}=\boldsymbol{Q}^{-1} \boldsymbol{x}$, where $\boldsymbol{Q}$ is the eigenvector matrix of $\hat{\boldsymbol{A}}_{\boldsymbol{M}}$ such that the state space can be separated into $\tilde{\boldsymbol{x}}=\left[\boldsymbol{x}_{\boldsymbol{d}}^{T}, \boldsymbol{x}_{\boldsymbol{i}}^{T}\right]^{T}$ with dynamical states $\boldsymbol{x}_{\boldsymbol{d}} \in \mathbb{R}^{n-n_{i}}$ and integrator states $\boldsymbol{x}_{\boldsymbol{i}} \in \mathbb{R}^{n_{i}}$. The dynamics of this transformed system are

$$
\begin{gathered}
\dot{\boldsymbol{x}}_{\boldsymbol{d}}=\boldsymbol{A}_{\boldsymbol{d}} \boldsymbol{x}_{\boldsymbol{d}}+\boldsymbol{B}_{\boldsymbol{d}} \ln \left(1+\frac{\tilde{\boldsymbol{c}}}{\boldsymbol{c}_{\boldsymbol{0}}}\right)+\boldsymbol{B}_{\boldsymbol{d}, \boldsymbol{i}} i(t) \\
y_{d}(t)=\boldsymbol{C}_{\boldsymbol{d}} \boldsymbol{x}_{\boldsymbol{d}}+\boldsymbol{D}_{\boldsymbol{d}} \ln \left(1+\frac{\tilde{\boldsymbol{c}}}{\boldsymbol{c}_{\boldsymbol{0}}}\right)+\boldsymbol{D}_{\boldsymbol{d}, \boldsymbol{i}} i(t) \\
\tilde{\boldsymbol{c}}=\boldsymbol{C}_{\boldsymbol{c}} \boldsymbol{x}=\left[\begin{array}{ll}
\boldsymbol{C}_{\boldsymbol{c}, \boldsymbol{1}} & \boldsymbol{C}_{\boldsymbol{c}, \boldsymbol{2}}
\end{array}\right]\left[\begin{array}{l}
\boldsymbol{x}_{\boldsymbol{d}} \\
\boldsymbol{x}_{\boldsymbol{i}}
\end{array}\right]=\boldsymbol{C}_{\boldsymbol{c}, \mathbf{1}} \boldsymbol{x}_{\boldsymbol{d}} \\
\dot{\boldsymbol{x}}_{i}=\boldsymbol{B}_{\boldsymbol{i}} i(t) \\
y_{i}(t)=\boldsymbol{C}_{\boldsymbol{i}} \boldsymbol{x}_{\boldsymbol{i}} \\
v(t)=y_{d}(t)+y_{i}(t) .
\end{gathered}
$$

Evaluating the integrator of (14a) means that (13c) can be expressed as

$$
\tilde{\boldsymbol{c}}=\boldsymbol{C}_{\boldsymbol{c}} \tilde{\boldsymbol{x}}=\boldsymbol{C}_{\boldsymbol{c}, \mathbf{1}} \boldsymbol{x}_{\boldsymbol{d}}+\boldsymbol{C}_{\boldsymbol{c}, \mathbf{2}} \boldsymbol{B}_{\boldsymbol{i}} \int_{0}^{t} i(\tau) d \tau .
$$

For supercapacitors, the matrices $\boldsymbol{C}_{\boldsymbol{c}, \mathbf{2}} \boldsymbol{B}_{\boldsymbol{i}}=0$, so the concentration can be expressed solely in terms of the dynamical states and independently of the integral of the current, i.e. $\tilde{\boldsymbol{c}}=\boldsymbol{C}_{\boldsymbol{c}, \mathbf{1}} \boldsymbol{x}_{\boldsymbol{d}}$. This means that the integrator states are unobservable with respect to the concentration, so there is not a unique mapping $\tilde{\boldsymbol{c}} \rightarrow \boldsymbol{x}_{\boldsymbol{i}}$. The independence of $\tilde{\boldsymbol{c}}$ with respect to the integral of $i(t)$ means that the supercapacitor model can be expressed in the form of Figure 3 with $\overline{\mathcal{I O}}$ mapping $\{\boldsymbol{\psi}(\boldsymbol{q}), i\} \rightarrow\left\{\boldsymbol{q}, y_{d}\right\}$ via (13) with $\boldsymbol{q}=\tilde{\boldsymbol{c}} / c_{0}$.

The upper subsystem of Figure 3 is labelled the integrator subsystem and the lower system involving $\overline{\mathcal{I O}}$ and the feedback loop of $\boldsymbol{\psi}(\boldsymbol{q})$ is labelled the dynamical subsystem. The independence on $\tilde{\boldsymbol{c}}$ from the integral of $i(t)$ greatly simplifies the stability analysis of the following section, as the dynamical and integrator systems can be decoupled and analysed separately. As such, the energy dissipation properties of the nonlinear dynamical subsystem can be analysed using absolute stability theory and by considering signals in $\mathcal{L}_{2}$.

A physical reason for the independence of the integrator dynamics is now given by returning to the underlying PDEs of the model. The model has two sets of dynamics; those for the concentration (2a) and those for the potentials $(2 \mathrm{~b})$. It is now shown that the integrator dynamics come from the electropotential dynamics (2b) and not from (2a). By differentiating with respect to space the expression for Ohm's law in the solid phase [48]

$$
\sigma \frac{\partial \phi_{s}(\chi, t)}{\partial \chi}=i(t)-i_{2}(\chi, t)
$$




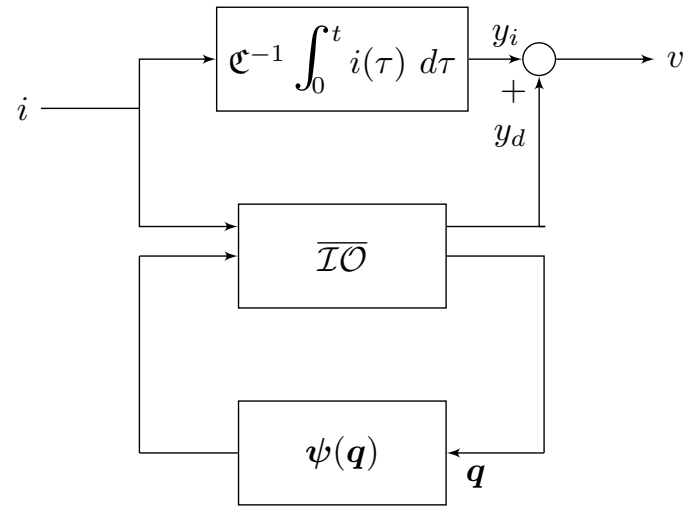

Figure 3: The reformulated supercapacitor model loop structure with the separation of the integrator and dynamical subsystems. The top linear system, known as the integrator subsystem, integrates the current $i$ giving a voltage $y_{i}$ with $\mathfrak{C}^{-1}$ being the inverse of a capacitance. The lower subsystem, denoted as the dynamical subsystem, is formed of the feedback interconnection of a linear mapping $\overline{\mathcal{I O}}$ from the logarithmic nonlinearities $\boldsymbol{\psi}(\boldsymbol{q})$ and current density $i$ to the ionic concentration deviation $\boldsymbol{q}=\tilde{\boldsymbol{c}} / c_{0}$ and a voltage $y_{d}$ to the nonlinearities. The voltage of the device $v$ is the sum of the voltages from the integrator and the dynamical subsystems.

where $i_{2}(\chi, t)$ is the current carried by the electrolyte, the dynamics of the potential difference across the double layer (2b) can be expressed as

$$
a C \frac{\partial\left(\phi_{s}(\chi, t)-\phi_{e}(\chi, t)\right)}{\partial t}=\frac{\partial i_{2}(\chi, t)}{\partial \chi}
$$

Integrating (18) with respect to space across an electrode and noting that all the current is carried in the solid phase at the current collector boundary $\left(i_{2}(0, t)=i_{2}(L, t)=\right.$ $0)$ and at the separator all the current is carried by the electrolyte phase $\left(i_{2}\left(L_{\text {elec }}, t\right)=i_{2}\left(L_{\text {elec }}+L_{\text {sep }}, t\right)=i(t)\right)$ [48] gives

$$
\begin{aligned}
& a C \frac{\partial}{\partial t} \int_{0}^{L_{e l e c}}\left(\phi_{s}(\chi, t)-\phi_{e}(\chi, t)\right) d \chi=-i(t), \\
& a C \frac{\partial}{\partial t} \int_{L_{e l e c}+L_{s e p}}^{L}\left(\phi_{s}(\chi, t)-\phi_{e}(\chi, t)\right) d \chi=i(t) .
\end{aligned}
$$

A set of integrator states is obtained from (19), with these states being the total potential difference across the double layer. By contrast, the diffusion operator in (2a) means that the concentration dynamics are not pure integrators. This agrees with the observation that $\boldsymbol{C}_{\boldsymbol{c}, \mathbf{2}} \boldsymbol{B}_{\boldsymbol{i}}=0$ implying that the dynamics of the concentration are independent of the integral of the current. Interestingly, (19) can be recognised as the typical dynamics of a capacitor consisting of two flat plates separated by an insulator.

The presence of an integrator makes physical sense for energy storage devices as these devices absorb the energy supplied by the external current and in theory store this energy indefinitely. In reality, supercapacitors exhibit a small leakage current that dissipates the stored energy over time, but this phenomenon, which is due to electric field relaxation in the double layer, was not included in the model.

\section{System Properties}

In this section, bounds on the system properties for the PB model (13) are computed, including model stability, the reachable set with unit energy impulse current, stored and dissipated energies for a charging profile, gains from current to voltage corresponding to the capacitance and resistance and the maximum voltage without the potential difference across the double layer exceeding the electrolyte overpotential.

The discretised version of the dynamic state-space model (13) can be considered as a Lurie system. Using the notation of [4], the Lurie system has the form

$$
\begin{aligned}
\dot{\boldsymbol{x}}_{\boldsymbol{d}} & =\boldsymbol{A} \boldsymbol{x}_{\boldsymbol{d}}+\boldsymbol{B}_{\boldsymbol{p}} \boldsymbol{p}+\boldsymbol{B}_{\boldsymbol{w}} w(t) \\
\boldsymbol{p}_{\boldsymbol{j}}(t) & =\boldsymbol{\psi}_{\boldsymbol{j}}\left(\boldsymbol{q}_{\boldsymbol{j}}(t)\right), \quad \boldsymbol{j}=1, \ldots, n_{p} \\
\boldsymbol{q} & =\boldsymbol{C}_{\boldsymbol{q}} \boldsymbol{x}_{\boldsymbol{d}} \\
z(t) & =\boldsymbol{C}_{\boldsymbol{z}} \boldsymbol{x}_{\boldsymbol{d}}+\boldsymbol{D}_{\boldsymbol{p}} \boldsymbol{p}+\boldsymbol{D}_{\boldsymbol{w}} w(t) .
\end{aligned}
$$

where $\boldsymbol{A}=\boldsymbol{A}_{\boldsymbol{d}}$ is Hurwitz, $\boldsymbol{B}_{\boldsymbol{p}}=\boldsymbol{B}_{\boldsymbol{d}}, \boldsymbol{B}_{\boldsymbol{w}}=\boldsymbol{B}_{\boldsymbol{d}, \boldsymbol{i}}$, $\boldsymbol{C}_{\boldsymbol{z}}=\boldsymbol{C}_{\boldsymbol{d}}, \boldsymbol{D}_{\boldsymbol{w}}=\boldsymbol{D}_{\boldsymbol{d}, \boldsymbol{i}}, w(t)=i(t) \in \mathbb{R}$ is the external input, $n_{p}$ is the number of Lurie system outputs, $z(t)=y_{d}(t) \in \mathbb{R}$ from (13b) is the dissipated voltage output, $\boldsymbol{q}=\tilde{\boldsymbol{c}} / c_{0} \in \mathbb{R}^{n_{p}}$ which includes boundary values. The nonlinearity $\boldsymbol{\psi}(\boldsymbol{q})=\ln (1+\boldsymbol{q}): \mathbb{R}^{n_{p}} \rightarrow \mathbb{R}^{n_{p}}$ is static, memoryless, locally Lipschitz in $\boldsymbol{q}$, decentralised with each $\boldsymbol{\psi}_{\boldsymbol{i}}\left(\boldsymbol{q}_{\boldsymbol{j}}\right)$ mapping an individual $\boldsymbol{q}_{\boldsymbol{j}}$ as in

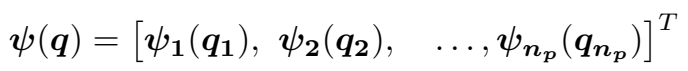

and locally sector bounded

$$
\frac{\boldsymbol{\psi}_{\boldsymbol{j}}\left(\boldsymbol{q}_{\boldsymbol{j}}\right)}{\boldsymbol{q}_{\boldsymbol{j}}} \in\left[\underline{\boldsymbol{\delta}_{\boldsymbol{j}}}, \overline{\boldsymbol{\delta}_{\boldsymbol{j}}}\right] \quad \forall \boldsymbol{q} \in \mathcal{Q} \subseteq \mathbb{R}^{n_{p}}
$$

with $0 \leq \boldsymbol{\delta}_{\boldsymbol{j}} \leq \overline{\boldsymbol{\delta}_{j}}$.

The shifted logarithmic nonlinearity of Fig. 4 is an unbounded nonlinearity, i.e. $\overline{\boldsymbol{\delta}_{j}} \rightarrow \infty$ as $\tilde{\boldsymbol{c}}_{j} \rightarrow-c_{0}$. To make the Lyapunov analysis of the following section tractable, the sector $\overline{\boldsymbol{\delta}}_{\boldsymbol{j}}$ is constrained to a finite value with the level sets of the Lyapunov function being contained within the set detailed by the sector condition $\mathcal{Q}=\left\{\boldsymbol{q}: \underline{\boldsymbol{q}}_{\boldsymbol{j}}<\boldsymbol{q}_{\boldsymbol{j}}<\overline{\boldsymbol{q}}_{\boldsymbol{j}}, \boldsymbol{j}=1, \ldots, n_{p}\right\}$ where $\underline{\boldsymbol{q}}_{\boldsymbol{j}}>-1$ and $\overline{\boldsymbol{q}}_{j}<\infty$. This means the analysis is only appropriate for a finite domain where $\boldsymbol{q} \in \mathcal{Q}$ [21]. The set of values of $\boldsymbol{x}_{\boldsymbol{d}}$ for which the local conditions hold is defined as $\mathcal{X}_{q}=\left\{\boldsymbol{x}_{\boldsymbol{d}}: \boldsymbol{q}\left(\boldsymbol{x}_{\boldsymbol{d}}\right) \in \mathcal{Q}\right\}$.

In order to compute bounds for the system properties, a positive definite Lyapunov function $V\left(\boldsymbol{x}_{\boldsymbol{d}}(t)\right)>0 \forall \boldsymbol{x}_{\boldsymbol{d}} \neq 0$ whose temporal derivative is negative definite

$$
\frac{d V\left(\boldsymbol{x}_{\boldsymbol{d}}(t)\right)}{d t}<0
$$

will be defined. In this paper, the Lurie-Postnikov Lyapunov function $[4]$

$$
V(\boldsymbol{\theta}(t))=\boldsymbol{\theta}(t)^{T} \boldsymbol{P} \boldsymbol{\theta}(t)+\sum_{\boldsymbol{j}=1}^{n_{p}} \boldsymbol{\lambda}_{\boldsymbol{j}} \int_{0}^{\boldsymbol{C}_{\boldsymbol{q}, \boldsymbol{j}} \boldsymbol{\theta}(t)} \boldsymbol{\psi}_{\boldsymbol{j}}(v) d v
$$




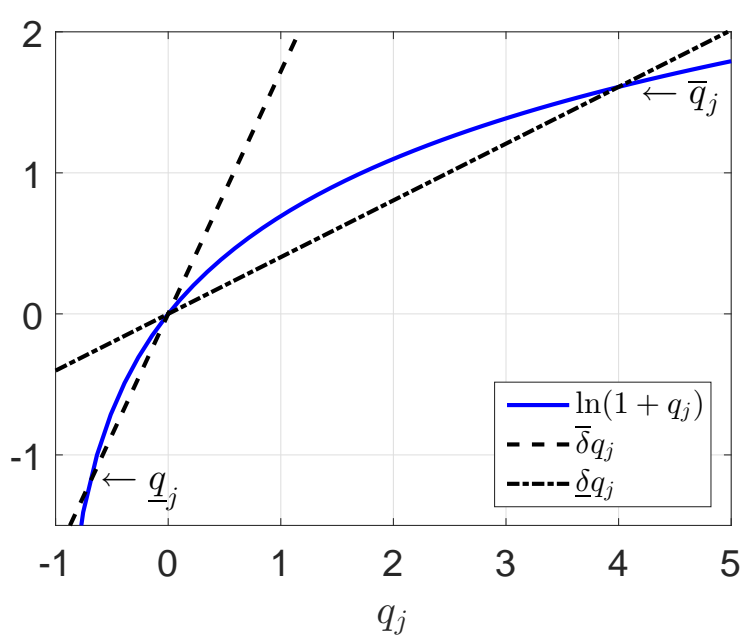

Figure 4: The locally sector bounded nonlinearity $\boldsymbol{\psi}\left(\boldsymbol{q}_{\boldsymbol{j}}\right)=$ $\ln \left(1+\boldsymbol{q}_{\boldsymbol{j}}\right)$. The Lyapunov analysis is valid locally when $\underline{\boldsymbol{q}}_{j} \leq \boldsymbol{q}_{j} \leq \overline{\boldsymbol{q}}_{j}$.

will be used where $\boldsymbol{P}>0, \boldsymbol{\lambda}_{\boldsymbol{j}} \geq 0$ and $\boldsymbol{C}_{\boldsymbol{q}, \boldsymbol{j}}$ denotes the $\boldsymbol{j}^{\text {th }}$ row of $\boldsymbol{C}_{q}$ [4]. The matrix form of (23) with this choice of Lyapunov function is given in (25).

Due to the sector conditions only holding locally, a local analysis is required. Classical results on the local analysis of Lurie system were developed for saturated systems in $[17,5]$, with more recent results given in [46, 47] and [13] which uses dissipation inequalities to give a local analysis using Zames-Falb multipliers. These results form the basis of the derivation presented here, which is applied to the Lyapunov function $V(\boldsymbol{\theta}(t))$, although, unlike [17], this approach allows both asymmetric domains and generic level sets to be considered. When $\mathbf{q} \in \mathcal{Q}$, the following inequality is satisfied

$$
\boldsymbol{\beta}_{\boldsymbol{j}}\left(\overline{\boldsymbol{q}}_{\boldsymbol{j}}-\boldsymbol{q}_{\boldsymbol{j}}\right)\left(\boldsymbol{q}_{\boldsymbol{j}}-\underline{\boldsymbol{q}}_{\boldsymbol{j}}\right) \geq 0, \quad \boldsymbol{j}=1, \ldots n_{p}
$$

with $\boldsymbol{\beta}_{\boldsymbol{j}}>0$. If also the following inequality holds

$$
\boldsymbol{\beta}_{\boldsymbol{j}}\left(\overline{\boldsymbol{q}}_{\boldsymbol{j}}-\boldsymbol{q}_{\boldsymbol{j}}\right)\left(\boldsymbol{q}_{\boldsymbol{j}}-\underline{\boldsymbol{q}}_{\boldsymbol{j}}\right) \geq \boldsymbol{\alpha}_{\boldsymbol{j}}-V\left(\boldsymbol{x}_{\boldsymbol{d}}(t)\right), \quad \boldsymbol{j}=1, \ldots n_{p}
$$

then the sublevel sets of $V\left(\boldsymbol{x}_{\boldsymbol{d}}(t)\right)$ defined by $\mathcal{E}(V, \underline{\alpha})$ where $\mathcal{E}(V, \underline{\alpha})=\left\{\boldsymbol{x}_{\boldsymbol{d}} \in \mathbb{R}^{n-n_{i}} \mid \underline{\alpha}-V\left(\boldsymbol{x}_{\boldsymbol{d}}(t)\right) \geq 0\right\}$ and $\underline{\alpha}=\min _{\boldsymbol{j}=1, \ldots, n_{p}} \boldsymbol{\alpha}_{\boldsymbol{j}}$ are contained within the local domain with $\mathcal{E}(V, \underline{\alpha}) \subseteq \mathcal{Q}$.

To obtain an LMI representation for the inclusion conditions of (27), a quadratic lower bound for $V\left(\boldsymbol{x}_{\boldsymbol{d}}\right)$ will be used

$$
\underline{V}(\boldsymbol{\theta}(t))=\boldsymbol{\theta}(t)^{T} \boldsymbol{P} \boldsymbol{\theta}(t) .
$$

Since $V\left(\boldsymbol{x}_{\boldsymbol{d}}(t)\right) \geq \underline{V}\left(\boldsymbol{x}_{\boldsymbol{d}}(t)\right)$ in the region where the sector conditions hold, if

$$
\boldsymbol{\beta}_{\boldsymbol{j}}\left(\overline{\boldsymbol{q}}_{\boldsymbol{j}}-\boldsymbol{q}_{\boldsymbol{j}}\right)\left(\boldsymbol{q}_{\boldsymbol{j}}-\underline{\boldsymbol{q}}_{\boldsymbol{j}}\right) \geq \boldsymbol{\alpha}_{\boldsymbol{j}}-\tilde{V}\left(\boldsymbol{x}_{\boldsymbol{d}}\right), \quad \boldsymbol{j}=1, \ldots n_{p}
$$

holds, then (27) holds with (29) being defined by the $n_{p}$
LMIs

$$
\begin{aligned}
\boldsymbol{\pi}_{j}= & {\left[\begin{array}{cc}
-\boldsymbol{\beta}_{j} \overline{\boldsymbol{q}}_{j} \underline{\boldsymbol{q}}_{j}-\boldsymbol{\alpha}_{j} & \frac{\boldsymbol{\beta}_{j}}{2}\left(\overline{\boldsymbol{q}}_{j}+\underline{\boldsymbol{q}}_{j}\right) C_{\boldsymbol{q}, \boldsymbol{j}} \\
\boldsymbol{C}_{\boldsymbol{q}, \boldsymbol{j}}^{\boldsymbol{T}}\left(\overline{\boldsymbol{q}}_{\boldsymbol{j}}+\underline{\boldsymbol{q}}_{j}\right) \frac{\boldsymbol{\beta}_{j}}{\mathbf{2}} & \boldsymbol{P}-\boldsymbol{\beta}_{j} C_{\boldsymbol{q}, \boldsymbol{j}}^{\boldsymbol{T}} C_{\boldsymbol{q}, \boldsymbol{j}}
\end{array}\right] \geq 0 . } \\
& \boldsymbol{j}=1, \ldots n_{p} .
\end{aligned}
$$

Satisfying these LMIs means that the local domain of the Lyapunov analysis is defined as $\mathcal{Q}_{0}=\mathcal{E}(\tilde{V}, \underline{\alpha})$ since $\mathcal{E}(\tilde{V}, \underline{\alpha}) \subseteq \mathcal{E}(V, \underline{\alpha}) \subseteq \mathcal{Q}$.

The time derivative of the Lyapunov function (24) has quadratic form given in (25) with $\boldsymbol{\Lambda}=\operatorname{diag}\left(\boldsymbol{\lambda}_{\mathbf{1}}, \ldots, \boldsymbol{\lambda}_{\boldsymbol{n}_{p}}\right) \geq 0$. The sector condition of (22) satisfies the inequality

$$
-\left(\boldsymbol{p}_{\boldsymbol{j}}-\underline{\boldsymbol{\delta}}_{\boldsymbol{j}} \boldsymbol{q}_{\boldsymbol{j}}\right)\left(\boldsymbol{p}_{\boldsymbol{j}}-\overline{\boldsymbol{\delta}}_{\boldsymbol{j}} \boldsymbol{q}_{\boldsymbol{j}}\right) \geq 0 \forall \boldsymbol{j}=1, \ldots, n_{p}, \forall \boldsymbol{q} \in \mathcal{Q}
$$

which is satisfied if

$$
\begin{aligned}
& s_{1}\left(x_{d}, p, T\right)= \\
& {\left[\begin{array}{ll}
x_{d}^{T} & p^{T}
\end{array}\right]\left[\begin{array}{cc}
-C^{T} \underline{\underline{\Delta}} \overline{T \Delta} C & C_{q}^{T} \underline{\underline{\Delta}+\bar{\Delta}} T \\
T \frac{\Delta+\bar{\Delta}}{2} C_{q} & -T
\end{array}\right]\left[\begin{array}{c}
x_{d} \\
p
\end{array}\right] \geq 0}
\end{aligned}
$$

where $\bar{\Delta}=\operatorname{diag}\left(\bar{\delta}_{1}, \ldots, \bar{\delta}_{n_{p}}\right), \underline{\Delta}=\operatorname{diag}\left(\underline{\delta}_{1}, \ldots, \underline{\delta}_{n_{p}}\right)$ and $\boldsymbol{T}=\operatorname{diag}\left(\boldsymbol{\tau}_{1}, \ldots, \boldsymbol{\tau}_{n_{p}}\right)>0$. Inequality constraints such as (25) and (32) are combined using the S-procedure $[4,19]$.

It is known that the Lyapunov function $V(\boldsymbol{\theta}(t))$ can give conservative solutions to absolute stability problems and that this conservatism can be reduced by relaxing the positivity of the multiplying scalars $\boldsymbol{\lambda}_{\boldsymbol{j}}$, such that they are sign-indefinite $[32,46]$, or by using slope information on the nonlinearity (with the shifted logarithm nonlinearity being locally slope restricted) [46]. In this paper, $V(\boldsymbol{\theta}(t))$ is used as: a) it is a classical function that is well understood [4] with the aim of this paper not being to propose new theoretical stability results, but instead to apply existing techniques to supercapacitor design, b) if the signs of $\boldsymbol{\lambda}_{\boldsymbol{j}}$ are relaxed from being positive then $\underline{V}(\boldsymbol{\theta}(t))$ is no longer a lower bound for $V(\boldsymbol{\theta}(t))$ which would complicate the local inclusion conditions of $(30), c)$ it was found that the added computational effort associated with using more advanced Lyapunov functions, such as the quadratic on $\boldsymbol{x}$ and $\boldsymbol{\psi}(\boldsymbol{q})$ Lyapunov function with sign indefinite $\boldsymbol{\lambda}_{j}$ [46], did not justify their reduced conservatism.

The following proposition gives a general semi-definite optimisation problem structure that allows the computations of the various system properties to be compactly described.

Proposition 1: Consider a cost function $\Pi$ to be minimised and a quadratic $\boldsymbol{M}=\dot{V}\left(\boldsymbol{x}_{\boldsymbol{d}}, \boldsymbol{p}, w\right)+s_{1}\left(\boldsymbol{x}_{\boldsymbol{d}}, \boldsymbol{p}, \boldsymbol{T}\right)+$ $\Omega(z(t), w(t))$ where $\dot{V}\left(\boldsymbol{x}_{\boldsymbol{d}}, \boldsymbol{p}, w\right)$ is the time derivative of $V\left(\boldsymbol{x}_{\boldsymbol{d}}(t)\right)$ along the trajectories of (20) as given in (25) and $\Omega(w, z)$ characterises a current/voltage property of interest. Feasible solutions to the semidefinite programme

$\min \Pi$

s.t. $\boldsymbol{P}>0, \boldsymbol{T}>0, \boldsymbol{M} \leq 0, \boldsymbol{\pi}_{\boldsymbol{j}} \geq 0$ for $\boldsymbol{j}=1, \ldots n_{p}$ 


$$
\dot{V}\left(\boldsymbol{x}_{\boldsymbol{d}}, \boldsymbol{p}, w(t)\right)=\left[\begin{array}{lll}
\boldsymbol{x}_{\boldsymbol{d}}^{T} & \boldsymbol{p}^{T} & w(t)
\end{array}\right]\left[\begin{array}{ccc}
\boldsymbol{A}^{T} \boldsymbol{P}+\boldsymbol{P} \boldsymbol{A} & \boldsymbol{P} \boldsymbol{B}_{\boldsymbol{p}}+\boldsymbol{A}^{T} \boldsymbol{C}_{\boldsymbol{q}}^{T} \boldsymbol{\Lambda} & \boldsymbol{P} \boldsymbol{B}_{\boldsymbol{w}} \\
\boldsymbol{B}_{\boldsymbol{p}}^{T} \boldsymbol{P}+\boldsymbol{\Lambda} \boldsymbol{C}_{\boldsymbol{q}} \boldsymbol{A} & \boldsymbol{\Lambda} \boldsymbol{C}_{\boldsymbol{q}} \boldsymbol{B}_{\boldsymbol{p}}+\boldsymbol{B}_{\boldsymbol{p}}^{T} \boldsymbol{C}_{\boldsymbol{q}}^{T} \boldsymbol{\Lambda} & \boldsymbol{\Lambda} \boldsymbol{C}_{\boldsymbol{q}} \boldsymbol{B}_{\boldsymbol{w}} \\
\boldsymbol{B}_{\boldsymbol{w}}^{T} \boldsymbol{P} & \boldsymbol{B}_{\boldsymbol{w}}^{T} \boldsymbol{C}_{\boldsymbol{q}}^{T} \boldsymbol{\Lambda} & 0
\end{array}\right]\left[\begin{array}{c}
\boldsymbol{x}_{\boldsymbol{d}} \\
\boldsymbol{p} \\
w(t)
\end{array}\right]<0
$$

give bounds on particular system properties of the supercapacitor model relating to stability and current/voltage criteria. Each of these criteria is discussed separately in the rest of this section. Standard semidefinite program solvers such as MOSEK [26] can be used to solve the optimisation problem. The formulations of these problems for the Lurie system are described in [4] with a key difference being that the analysis presented here is local.

\section{A. Case 1: Stability}

With $\Pi=\{\}, \Omega(z(t), w(t))=0$ and $w(t)=0$, the solution of Proposition 1 gives a sufficient condition on the local stability of the model. Increasing the bounds $\boldsymbol{q}$ and $\overline{\boldsymbol{q}}$, and hence the sector conditions, until no solution exists, defines the limits on how far the ionic concentration can theoretically be pushed during charging.

\section{B. Case 2: Maximum Reachable Set with Unit Energy Impulse}

With $\Pi=\operatorname{trace}(\boldsymbol{P}), \underline{\alpha}=1$ and $\Omega(z(t), w(t))=$ $-w(t)^{2}$, the solution of Proposition 1 gives an outer approximation to the local maximal reachable set with unit energy impulse of current $R_{u e} \triangleq\left\{\boldsymbol{x}_{\boldsymbol{d}}(t): \boldsymbol{x}_{\boldsymbol{d}}(0)=0, \int_{0}^{t} w(\tau)^{2} d \tau \leq 1\right\}$ for all $\left\{\boldsymbol{x}_{\boldsymbol{d}}, w\right\}$ satisfying (20),(29) and $\boldsymbol{x}_{\boldsymbol{d}}(t)$ being the value of $\boldsymbol{x}_{\boldsymbol{d}}$ at time $t$. The outer approximation of this set is defined by $\Xi=\left\{\boldsymbol{\xi}: \boldsymbol{\xi}^{T} \boldsymbol{P} \boldsymbol{\xi} \leq 1\right\}$ with $\Xi \supseteq R_{u e}$. The trajectories of the integrator subsystem can be obtained analytically by integrating (14a) such that

$$
\boldsymbol{x}_{\boldsymbol{i}}(t)-\boldsymbol{x}_{\boldsymbol{i}}(0)=\boldsymbol{B}_{\boldsymbol{i}} \int_{0}^{t} i(\tau) d \tau
$$

where $\boldsymbol{x}_{\boldsymbol{i}}(t)$ and $\boldsymbol{x}_{\boldsymbol{i}}(0)$ are the integrator states at times $t$ and 0 .

The physical interpretation of the set $R_{u e}$ is that it gives an upper bound for the maximum deviation from equilibrium of the concentration and potentials with the current supplying 1 Joule of energy. Input charging signals of arbitrary energy can similarly be analysed by scaling. Knowledge of this set is useful for safety purposes, i.e. when the physical states are required to remain within some limit when power is supplied to the supercapacitor.

\section{Case 3: Bounds on Stored and Dissipated Energy}

With $\Pi=\boldsymbol{x}_{\boldsymbol{d}}(0)^{T} \boldsymbol{P} \boldsymbol{x}_{\boldsymbol{d}}(0), w(t)=0, \underline{\alpha}=V\left(\boldsymbol{x}_{\boldsymbol{d}}(0)\right)$ and $\Omega(z(t), w(t))=z(t)^{2}$, the energy dissipated by the system in returning to the equilibrium from some initial condition $\boldsymbol{x}_{\boldsymbol{d}}(0) \in \mathcal{X}_{q}$

$$
J_{d}=\int_{0}^{\infty} z(\tau)^{2} d \tau
$$

is upper bounded by $\boldsymbol{x}_{\boldsymbol{d}}(0)^{T} \boldsymbol{P} \boldsymbol{x}_{\boldsymbol{d}}(0)$. No energy from the integrator subsystem is dissipated, so (35) defines the dissipated energy of the whole system. The energy stored in the integrator subsystem after a charge of time $t$ is

$$
J_{i}=\int_{0}^{t} i(\tau) d \tau \boldsymbol{B}_{\boldsymbol{i}}^{T} \boldsymbol{C}_{\boldsymbol{i}}^{T} \boldsymbol{C}_{\boldsymbol{i}} \boldsymbol{B}_{\boldsymbol{i}} \int_{0}^{t} i(\tau) d \tau .
$$

After charging, the energy stored in the supercapacitor is given by $J_{i}$ while the energy that is subsequently dissipated is upper bounded by $J_{d}$. Energy storage is achieved by the separation of the two electrodes by the electrically insulating separator, while the dissipated energy is related to the diffusion of the concentration. The stored energy can be drawn from the supercapacitor at any time and should be the property that is used to compare the energy storage performance of different supercapacitors. The dissipated energy is an often overlooked phenomenon and improvements to state-of-charge estimation and device safety can be made by considering its effect.

Figure 5 shows a simulation of the model with the parameters of a SAFT America supercapacitor obtained from [48] that are given in Table II. The charging profile is as follows; the supercapacitor is at rest for $5 \mathrm{~s}$ before a 100A constant current (CC) charge is applied for $23 \mathrm{~s}$, whereupon the current is removed and the device returns to an equilibrium. Experimental data for the $\mathrm{CC}$ charging period up to the point where the current was turned off, which was also obtained from [48], is shown. The upper bound on $J_{d}$ for the rest period after $25 \mathrm{~s}$ of the charge obtained by solving Proposition 1 was $1.46 \times 10^{-4}$ and the value obtained by numerically integrating the simulation output signal was $1.38 \times 10^{-4}$.

\section{Case 4: Capacitance and Resistance}

With $\Pi=\gamma_{d}^{2}, \underline{\alpha}=\|w\|_{2}$ and $\Omega(z(t), w(t))=z(t)^{2}$ $\gamma_{d}^{2} w(t)^{2}$, the $\mathcal{L}_{2}$ gain of the dynamic subsystem is upper bounded by $\gamma_{d}$. The gain $\gamma_{d}$ has the physical representation of bounding the resistance of the supercapacitor. The gain from $i$ to $\boldsymbol{y}_{\boldsymbol{i}}$ of the integrator subsystem is defined by

$$
\boldsymbol{y}_{\boldsymbol{i}}(t)-\boldsymbol{y}_{\boldsymbol{i}}(0)=\boldsymbol{C}_{\boldsymbol{i}} \boldsymbol{B}_{\boldsymbol{i}} \int_{0}^{t} i(\tau) d \tau
$$

where $\boldsymbol{y}_{\boldsymbol{i}}(t), \boldsymbol{y}_{\boldsymbol{i}}(0)$ are the values of $\boldsymbol{y}_{\boldsymbol{i}}$ at times $t$ and 0 . Defining the capacitance $\mathfrak{C}$ by $Q=\mathfrak{C} v_{s}$ where $Q=$ $\int_{0}^{t} i(\tau) d \tau$ is the transferred charge and $v_{s}(t)=\boldsymbol{y}_{\boldsymbol{i}}(t)-$ $\boldsymbol{y}_{\boldsymbol{i}}(0)$ is the voltage stored, the capacitance can be represented by $\mathfrak{C}=\left(\boldsymbol{C}_{\boldsymbol{i}} \boldsymbol{B}_{\boldsymbol{i}}\right)^{-1}$ upon consideration of (37).

One of the main advantages of EC models is that expressions for their capacitance and resistance can be easily obtained by resolving the circuit. Obtaining bounds for these electrical properties as functions of the physical 


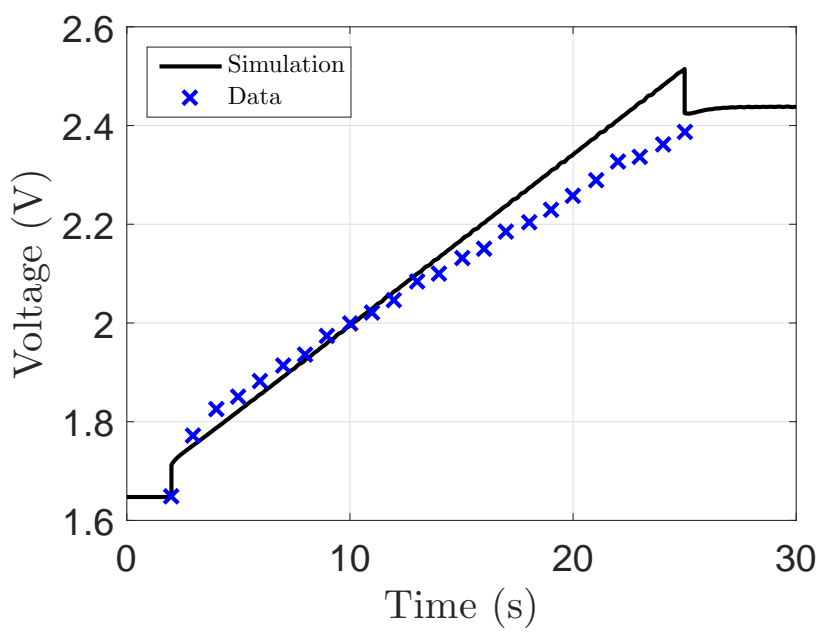

Figure 5: Simulation results and experimental data of the SAFT America supercapacitor. The data and the model parameters were obtained from [48]. The supercapacitor was rested for $5 \mathrm{~s}$ before a $100 \mathrm{~A}$ current was applied for 23s. After this charge, the current was removed and the voltage returned to an equilibrium.

parameters provides a design method for constructing supercapacitors with certain resistance and capacitance properties.

Figure 6 a shows experimental data and simulation results of the voltage response of a supercapacitor from Maxwell Technologies with part number P0100 P270T01, when subjected to the square wave charging profile of Figure $6 \mathrm{~b}$. The model parameters values are given in Table II. The experimental data was obtained from [11] and the bounds $\overline{\boldsymbol{q}}, \boldsymbol{q}$ characterising the local domain were obtained from the simulation. The $\mathcal{L}_{2}$ gain from the simulation was $\gamma_{d, \text { sim }}=0.3065$ while the upper bound obtained by computing Proposition 1 with a 15 state discretisation of (2) was $\gamma_{d}=0.3108$. The value of the capacitance was $\mathfrak{C}=1 / 0.86=1.16$.

The conservatism of the computed bound for $\gamma_{d}$ is due to the inherent conservatism of absolute stability theory and the satisfaction of the local inclusion conditions. Increasing the limits $\overline{\boldsymbol{q}}$ and $\boldsymbol{q}$ where the local sector conditions hold leads to a larger sector condition that gives a less accurate representation of the logarithm, and hence increases conservatism. However, increasing the bounds also leads to less stringent conditions on the geometry of the Lyapunov function imposed by the local inclusion conditions of (30), reducing conservatism.

The variation of the the resistance $\gamma_{d}$ and capacitance $\mathfrak{C}$ as functions of the electrode length $L_{\text {elec }}$ are shown in Figure 7 for the Maxwell Technologies supercapacitor. This figure shows conflicting design objectives have to be considered when designing supercapacitors, with devices that have low resistances but high capacitances being desired.

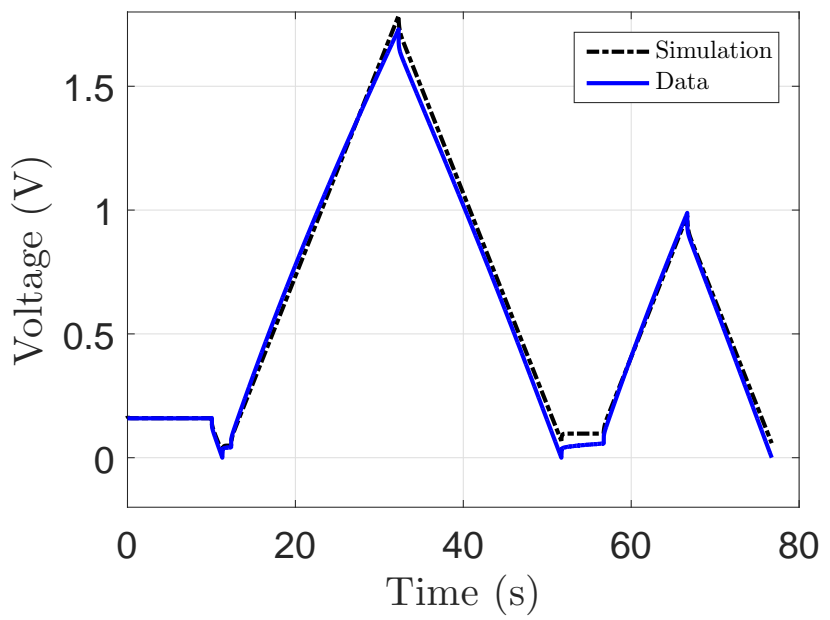

(a) Voltage response.

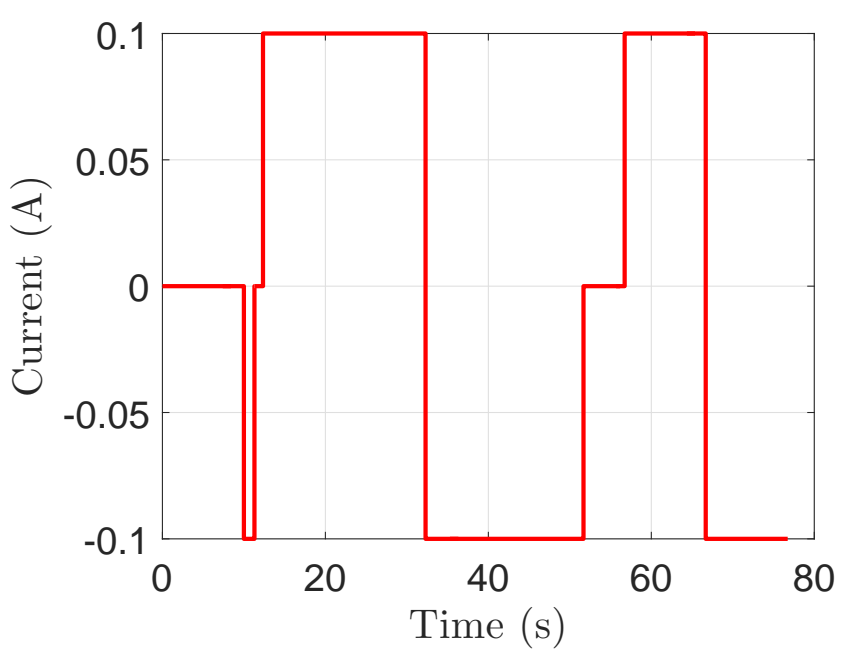

(b) Current profile.

Figure 6: Simulation results and experimental data from [11] for the square wave charging of the Maxwell Technologies supercapacitor.

\section{E. Maximum Voltage Limits}

Finally, an upper bound on the supercapacitor voltage is computed such that the potential difference across the double layer does not exceed the overpotential [23]. The main limitation holding back the implementation of supercapacitors is their low energy densities, defined as

$$
E=\frac{1}{2} \mathfrak{C} v^{2}
$$

Low maximum operating voltages, enforced to prevent decomposition of the electrolyte, are one of the reasons why this energy density is low [23]. For aqueous electrolytes, such as that used in [48], if the potential difference between the electrode and the solvated ion is greater than $1.27 \mathrm{~V}$, then the solvated ions undergo the overall reaction

$$
2 \mathrm{H}_{2} \mathrm{O}(\mathrm{l}) \rightarrow 2 \mathrm{H}_{2}(\mathrm{~g})+\mathrm{O}_{2}(\mathrm{~g})
$$




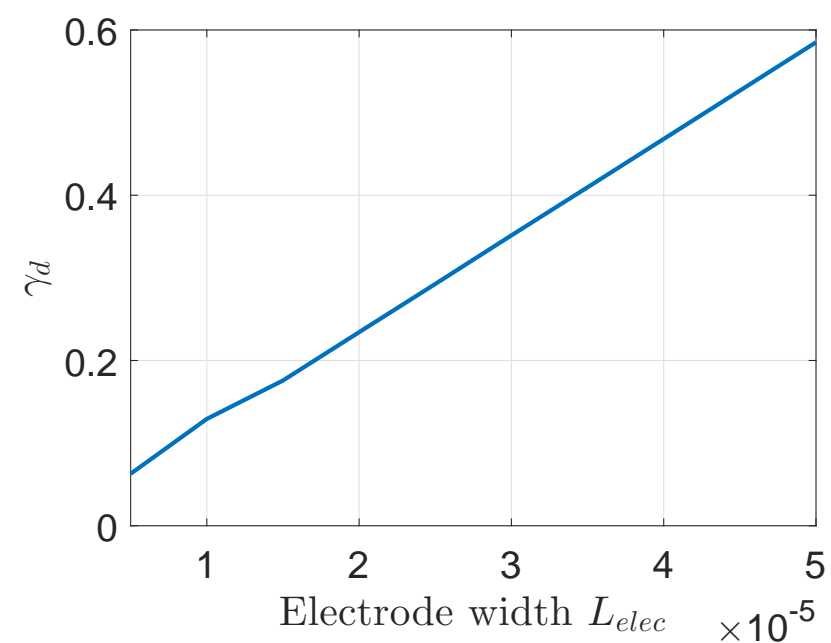

(a) Gain of the dynamic subsystem describing resistance.

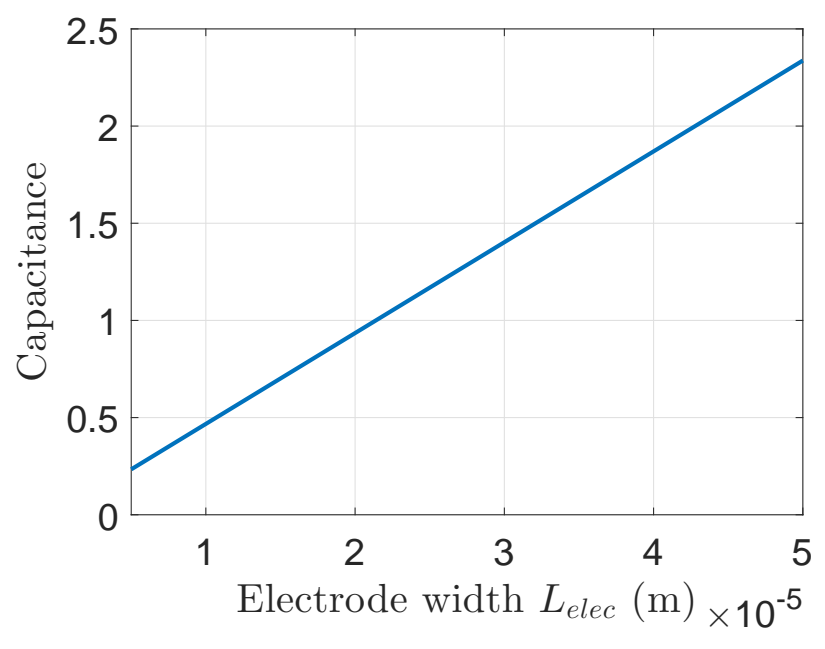

(b) Capacitance.

Figure 7: Variation in the $\mathcal{L}_{2}$ gain of the dynamical subsystem, describing resistance, and the capacitance as functions of the electrode lengths for the Maxwells Technologies supercapacitor.

transitioning from a liquid to a gas. This causes the charges in the electrolyte and the electrode to no longer be separated and a short circuit occurs.

To compute this maximum voltage, the term $\kappa \partial \phi_{s}(\chi, t) / \partial \chi$ is added and subtracted to the algebraic constraint $(2 \mathrm{c})$ such that it can be expressed as

$$
\begin{aligned}
\frac{\partial \phi_{s}(\chi, t)}{\partial \chi}= & -\frac{\kappa}{\sigma+\kappa} \frac{\partial\left(\phi_{e}(\chi, t)-\phi_{s}(\chi, t)\right)}{\partial \chi} \\
& -\frac{\kappa\left(t_{+}-t_{-}\right)}{f(\kappa+\sigma)} \frac{\partial \ln (c(\chi, t))}{\partial \chi}-\frac{i(t)}{\sigma+\kappa}
\end{aligned}
$$

Substituting this expression into the definition of the

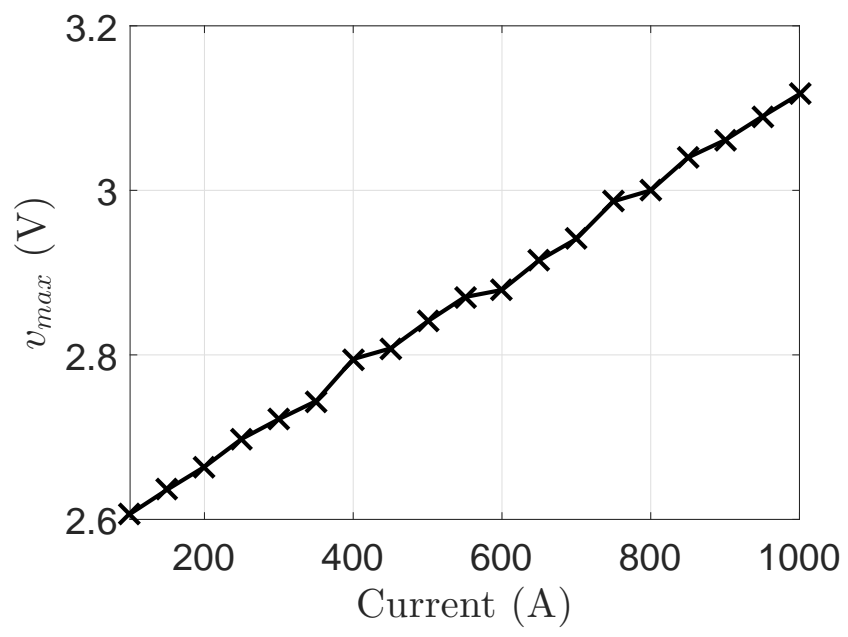

Figure 8: The maximum voltage of the SAFT America supercapacitor such that potential across the double layer never exceeds the overpotential as a function of current $I$.

voltage from (3) gives

$$
\begin{aligned}
v= & \phi_{s}(0, t)-\phi_{s}(L, t) \\
= & -\int_{0}^{L} \frac{\partial \phi_{s}(\chi, t)}{\partial \chi} d \chi \\
= & \underbrace{\left.\frac{\kappa}{\sigma+\kappa}\left(\phi_{e}(\chi, t)-\phi_{s}(\chi, t)\right)\right|_{\chi=0} ^{\chi=L}}_{(a)}+\underbrace{\frac{2 i(t) L_{\text {elec }}}{\sigma+\kappa}}_{(b)} \\
& +\underbrace{\left.\frac{\kappa\left(t_{+}-t_{-}\right)}{f(\kappa+\sigma)} \ln (c(\chi, t))\right|_{\chi=0} ^{\chi=L}}_{(c)} .
\end{aligned}
$$

This algebraic relation for the voltage expressed in terms of the boundary values of the states has been split into three components: (a) the potential difference across the double layer, (b) the series resistance term and (c) the charge carried by the ions. This expression shows that the voltage is linearly dependent upon the maximum potential difference across the double layer and the current $i$. The maximum value of the logarithmic term in (42c) depends upon the extremal values of the interval $c(\chi, t) \in[\underline{c}, \bar{c}]$, since the image of this set under the function $\ln (c(\chi, t))$ is concave when $\underline{c}>0$. The following optimisation problem computes the maximum voltage with a limit on the double layer potential difference

$$
v_{\max }=\max _{c(\chi, t), \phi_{s}(\chi, t), \phi_{e}(\chi, t)} v\left(c(\chi, t), \phi_{s}(\chi, t), \phi_{e}(\chi, t)\right)
$$

subject to $(9),\left|\phi_{s}(\chi, t)-\phi_{e}(\chi, t)\right| \leq 1.27, c(\chi, t) \in[\underline{c}, \bar{c}]$

$$
\text { and } B C s\left(c(\chi, t), \phi_{s}(\chi, t), \phi_{e}(\chi, t)\right) \text {. }
$$

To ensure that the optimisation searches over the physically real subset of the state-space, the algebraic equation (9) and state boundary conditions $B C s\left(c(\chi, t), \phi_{s}(\chi, t), \phi_{e}(\chi, t)\right)$, are included in (43) as 
equality constraints and an inequality condition is enforced to ensure that the potential difference across the double layer never exceeds $1.27 \mathrm{~V}$ at any point in the domain. This constrained, nonlinear optimisation problem can be solved for the discretised problem using standard MATLAB solvers such as "fmincon" and Figure 8 shows the variation of this maximum voltage as a function of the current $I$ for the case of the SAFT America supercapacitor. The quoted maximum voltage of the supercapacitor given by the manufacturers is $2.8 \mathrm{~V}$ [48]. The results of (43) are close to this manufacturing limit, however, it shows that this is a conservative limit as the maximum voltage is actually dependent upon the current profile, suggesting that more reasonable limits could be implemented for known charging conditions. It is pointed out that the PDE model does not have a detailed description of the double layer but instead uses porous electrode theory, which assumes that the electrode can be treated as a single homogenised phase. The double layer is a physical phenomenon that is not well understood, especially in complex geometries such as porous media [23], which means that the analysis of (43) may not capture local effects occurring in the double layer. The benefits of increasing the maximum voltage of the supercapacitor are significant as a doubling of the voltage results in a quadrupling of the maximum energy, as described by (38).

\section{F. Absolute Stability Theory for Energy Storage Devices}

Although this work has considered electrochemical supercapacitor models, the approach has many features that make it attractive for analysing and understanding other forms of electrochemical energy storage systems. Firstly, it is noted that absolute stability theory was partly inspired by the notion of passivity in circuit analysis, and the presented approach links these two classic approaches through the electrochemistry. Robustness is a core concept in absolute stability theory, which can accommodate the approximations and parameterisations of the complex PB electrochemical models. This robustness comes at the expense of conservatism that could be overcome by using more advanced Lyapunov functions [46]. The analysis could also be extended to time-varying systems, allowing supercapacitor degradation to be considered. Lastly, since most energy storage devices are known to be passive, the proposed approach can be applied to models of other devices such as the Newman model for Li-ion batteries [9] whose main nonlinearities are the OCV curve and the Butler Volmer equation, which can both be represented by sector conditions.

\section{Conclusion}

Bounds for the system properties of a physics-based supercapacitor model have been computed in this paper. By treating the model as a Lurie system, bounds for the dissipated and stored energies after charging, gains from current to voltage corresponding to the capacitance and the resistance, model stability, maximum reachable set with unit energy impulse current and limits on the maximum voltage such that the potential difference across the double layer never exceeded the electrolyte overpotential were computed. These bounds were obtained by solving several optimisation problems involving linear matrix inequalities and semi-definite programmes.

\section{ACKNOWLEDGMENT}

The authors are grateful to the anonymous reviewers for their insightful comments and suggestions.

\section{APPENDIX}

The matrices forming the discretised version of the electrochemical supercapacitor model are

$$
\begin{aligned}
\boldsymbol{M} & =\left[\begin{array}{cc}
\boldsymbol{M}_{\mathbf{1 1}} & \boldsymbol{M}_{\mathbf{1 2}} \\
\mathbf{0} & \boldsymbol{M}_{\mathbf{2 2}}
\end{array}\right]=\left[\begin{array}{cc}
\epsilon \boldsymbol{I} & \frac{a C}{F}\left(t_{-} \frac{d q_{+}}{d q}+t_{+} \frac{d q_{-}}{d q}\right) \boldsymbol{I} \\
0 & a C \boldsymbol{I}
\end{array}\right] \\
\hat{\boldsymbol{A}} & =\left[\begin{array}{cc}
\hat{\boldsymbol{A}}_{\mathbf{1 1}} & \mathbf{0} \\
\mathbf{0} & \hat{\boldsymbol{A}}_{\mathbf{2 2}}
\end{array}\right] \\
& =\left[\begin{array}{cc}
D \hat{\mathbf{D}}_{c}^{2} & \\
0 & \sigma \hat{\mathbf{D}}_{\phi_{s}}^{2}\left(I-\left(\kappa \hat{\mathbf{D}}_{\phi_{e}}+\sigma \hat{\mathbf{D}}_{\phi_{s}}\right)^{-1} \sigma \hat{\mathbf{D}}_{\phi_{s}}\right)
\end{array}\right]
\end{aligned}
$$

$$
\begin{aligned}
& \hat{\boldsymbol{B}}=\left[\begin{array}{c}
0 \\
\hat{\boldsymbol{B}}_{1}
\end{array}\right]
\end{aligned}
$$

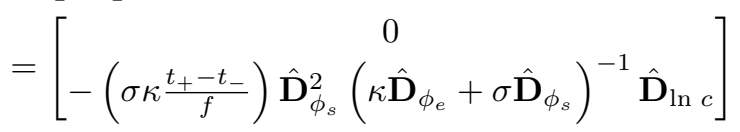

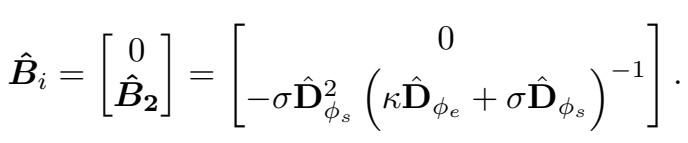

\section{REFERENCES}

[1] M. Al-Sakka, H. Gualous, J. V. Mierlo, and H. Culcu, "Thermal modeling and heat management of supercapacitor modules for vehicle applications," Journal of Power Sources, vol. 194, no. 2, pp. 581-587, 2009.

[2] S. Allu, B. Velamur Asokan, W. A. Shelton, B. Philip, and S. Pannala, "A generalized multi-dimensional mathematical model for charging and discharging processes in a supercapacitor," Journal of Power Sources, vol. 256, pp. 369 - 382, 2014.

[3] A. M. Bizeray, S. Zhao, S. R. Duncan, and D. A. Howey, "Lithium-ion battery thermal-electrochemical model-based state estimation using orthogonal collocation and a modified extended Kalman filter," Journal of Power Sources, vol. 296, pp. 400-412, 2015.

[4] S. Boyd, L. E. Ghaoui, E. Feron, V. Balakrishnan, and V. A. Yakubovich, Linear matrix inequalities in system and control theory. Philadelphia, PA, USA: SIAM, 1994.

[5] J. M. G. Da Silva and S. Tarbouriech, "Local stabilization of discrete-time linear systems with saturating 
controls: An LMI-based approach," IEEE Transactions on Automatic Control, vol. 46, no. 1, pp. 119$125,2001$.

[6] Y. Dai and V. Srinivasan, "On graded electrode porosity as a design tool for improving the energy density of batteries," Journal of the Electrochemical Society, vol. 163, no. 3, pp. A406-A416, 2016.

[7] S. De, P. W. C. Northrop, V. Ramadesigan, and V. R. Subramanian, "Model-based simultaneous optimization of multiple design parameters for lithium-ion batteries for maximization of energy density," Journal of Power Sources, vol. 227, pp. 161-170, 2013.

[8] A. d'Entremont and L. Pilon, "First-principles thermal modeling of electric double layer capacitors under constant-current cycling," Journal of Power Sources, vol. 246, pp. 887-898, 2014.

[9] M. Doyle, T. Fuller, and J. Newman, "Modeling of galvanostatic charge and discharge of the lithium/polymer/insertion cell," Journal of the Electrochemical Society, vol. 140, no. 6, pp. 1526-1533, 1993.

[10] R. Drummond, D. A. Howey, and S. R. Duncan, "Loworder mathematical modelling of electric double layer supercapacitors using spectral methods," Journal of Power Sources, vol. 277, pp. 317-328, 2015.

[11] _ _ "Parameter estimation of an electrochemical supercapacitor model," in Proc. of the European Control Conference (ECC). Aarlberg, Denamrk, 2016, pp. 16.

[12] R. Drummond, S. Zhao, D. A. Howey, and S. R. Duncan, "Circuit synthesis of electrochemical supercapacitor models," Journal of Energy Storage, vol. 10, pp. 48-55, 2017.

[13] M. Fetzer and C. W. Scherer, "Zames-falb multipliers for invariance," IEEE Control Systems Letters, vol. 1, no. 2, pp. 412-417, 2017.

[14] T. F. Fuller, M. Doyle, and J. Newman, "Simulation and optimization of the dual lithium ion insertion cell," Journal of the Electrochemical Society, vol. 141, no. 1, pp. 1-10, 1994.

[15] S. Golmon, K. Maute, and M. L. Dunn, "A design optimization methodology for Li+ batteries," Journal of Power Sources, vol. 253, pp. 239-250, 2014.

[16] M. R. Hasyim, D. Ma, R. Rajagopalan, and C. Randall, "Prediction of charge-discharge and impedance characteristics of electric double-layer capacitors using porous electrode theory," Journal of The Electrochemical Society, vol. 164, no. 13, pp. A2899-A2913, 2017.

[17] H. Hindi and S. Boyd, "Analysis of linear systems with saturation using convex optimization," in Proc. of the Conference on Decision and Control. Tampa, Florida, USA, 1998, pp. 903-908.

[18] T. $\mathrm{Hu}$ and Z. Lin, Control systems with actuator saturation: analysis and design. Ann Arbor, MI, USA: Springer, 2001.

[19] U. Jönsson, "Lecture notes on integral quadratic constraints," KTH, Stockholm. ISBN 1401-2294, 2000.
[20] U. Jönsson and A. Megretski, "The Zames-Falb IQC for systems with integrators," IEEE Transactions on Automatic Control, vol. 45, no. 3, pp. 560-565, 2000.

[21] H. Khalil, Nonlinear Systems. Upper Saddle River, NJ, USA: Prentice Hall, 2002, vol. 3.

[22] M. Kroupa, G. J. Offer, and J. Kosek, "Modelling of supercapacitors: Factors influencing performance," Journal of The Electrochemical Society, vol. 163, no. 10, pp. A2475-A2487, 2016.

[23] M. Lu, F. Beguin, and E. Frackowiak, Supercapacitors: Materials, Systems and Applications. Weinheim, Germany: John Wiley \& Sons, 2013.

[24] X. Luo, J. Wang, M. Dooner, and J. Clarke, "Overview of current development in electrical energy storage technologies and the application potential in power system operation," Applied Energy, vol. 137, pp. 511-536, 2015.

[25] C. Monroe, M. Urbakh, and A. A. Kornyshev, "Double-layer effects in electrowetting with two conductive liquids," Journal of The Electrochemical Society, vol. 156, no. 1, pp. P21-P28, 2009.

[26] A. P. S. MOSEK, The MOSEK optimization toolbox for MATLAB manual. Version 7.1 (Revision 28)., 2015. [Online]. Available: http://docs.mosek.com/7.1/toolbox/index.html

[27] J. Newman, "Optimization of porosity and thickness of a battery electrode by means of a reaction-zone model," Journal of the Electrochemical Society, vol. 142, no. 1, pp. 97-101, 1995.

[28] J. Newman and K. E. Thomas-Alyea, Electrochemical systems. Hoboken, NJ, USA: John Wiley \& Sons, 2012.

[29] J. Newman and W. Tiedemann, "Porous-electrode theory with battery applications," AIChE Journal, vol. 21, no. 1, pp. 25-41, 1975.

[30] J. Newman, "FORTRAN programs for simulation of electrochemical systems," University of California, Berkley, CA, 2008.

[31] J. Niu, B. E. Conway, and W. G. Pell, "Comparative studies of self-discharge by potential decay and floatcurrent measurements at $\mathrm{C}$ double-layer capacitor and battery electrodes," Journal of Power Sources, vol. 135, no. 1, pp. 332-343, 2004.

[32] P. Park, "A revisited Popov criterion for nonlinear Lur'e systems with sector-restrictions," International Journal of Control, vol. 68, no. 3, pp. 461-470, 1997.

[33] V. Ramadesigan, R. N. Methekar, F. Latinwo, R. D. Braatz, and V. R. Subramanian, "Optimal porosity distribution for minimized ohmic drop across a porous electrode," Journal of The Electrochemical Society, vol. 157, no. 12, pp. A1328-A1334, 2010.

[34] V. Ramadesigan, P. W. C. Northrop, S. De, S. Santhanagopalan, R. D. Braatz, and V. R. Subramanian, "Modeling and simulation of lithium-ion batteries from a systems engineering perspective," Journal of The Electrochemical Society, vol. 159, no. 3, pp. R31R45, 2012.

[35] A. Rantzer, "On the Kalman-Yakubovich-Popov 
lemma," Systems \& Control Letters, vol. 28, no. 1, pp. 7-10, 1996.

[36] V. Răsvan, D. Danciu, and D. Popescu, "On absolute (robust) stability: Slope restrictions and stability multipliers," International Journal of Robust and Nonlinear Control, vol. 23, no. 1, pp. 77-103, 2013.

[37] D. B. Robinson, "Optimization of power and energy densities in supercapacitors," Journal of Power Sources, vol. 195, no. 11, pp. 3748-3756, 2010.

[38] A. Romero-Becerril and L. Alvarez-Icaza, "Reduced order dynamical model for supercapacitors," in Proc. rth IEEE International Conference on Electrical Engineering Computing Science and Automatic Control, Tuxtla Gutierrez, Mexico, 2010, pp. 71-76.

[39] P. Sharma and T. S. Bhatti, "A review on electrochemical double-layer capacitors," Energy Conversion and Management, vol. 51, no. 12, pp. 2901-2912, 2010.

[40] V. Srinivasan and J. W. Weidner, "Mathematical modeling of electrochemical capacitors," Journal of the Electrochemical Society, vol. 146, no. 5, pp. 16501658, 1999.

[41] B. Suthar, P. W. C. Northrop, R. D. Braatz, and V. R. Subramanian, "Optimal charging profiles with minimal intercalation-induced stresses for lithiumion batteries using reformulated pseudo 2-dimensional models," Journal of The Electrochemical Society, vol. 161, no. 11, pp. F3144-F3155, 2014.

[42] B. Suthar, P. W. C. Northrop, D. Rife, and V. R. Subramanian, "Effect of porosity, thickness and tortuosity on capacity fade of anode," Journal of The Electrochemical Society, vol. 162, no. 9, pp. A1708A1717, 2015.

[43] B. Suthar, V. Ramadesigan, S. De, R. D. Braatz, and V. R. Subramanian, "Optimal charging profiles for mechanically constrained lithium-ion batteries," Physical Chemistry Chemical Physics, vol. 16, no. 1, pp. 277-287, 2014.

[44] L. Trefethen, Spectral Methods in MATLAB. Philadelplhia, PA, USA: SIAM, 2000, vol. 10.

[45] U. Tröltzsch, O. Kanoun, and H.-R. Tränkler, "Characterizing aging effects of lithium ion batteries by impedance spectroscopy," Electrochimica Acta, vol. 51, no. 8, pp. 1664-1672, 2006.

[46] G. Valmorbida, R. Drummond, and S. R. Duncan, "Positivity conditions of Lyapunov functions for systems with slope restricted nonlinearities," in Proc. of the American Control Conference (ACC). Boston, USA, 2016, pp. 258-263.

[47] G. Valmorbida, A. Garulli, and L. Zaccarian, "Regional $\mathcal{L}_{2 m}$ gain analysis for linear saturating systems," Automatica, vol. 76, pp. 164-168, 2017.

[48] M. W. Verbrugge and P. Liu, "Microstructural analysis and mathematical modeling of electric doublelayer supercapacitors," Journal of the Electrochemical Society, vol. 152, no. 5, pp. D79-D87, 2005.

[49] J. Yan, Q. Wang, T. Wei, and Z. Fan, "Recent advances in design and fabrication of electrochemical supercapacitors with high energy densities," Advanced Energy Materials, vol. 4, no. 4, 2014.

[50] G. Zames and P. Falb, "Stability conditions for systems with monotone and slope-restricted nonlinearities," SIAM Journal on Control, vol. 6, no. 1, pp. 89-108, 1968.

[51] L. Zhang, Z. Wang, X. Hu, F. Sun, and D. G. Dorrell, "A comparative study of equivalent circuit models of ultracapacitors for electric vehicles," Journal of Power Sources, vol. 274, pp. 899-906, 2015. 